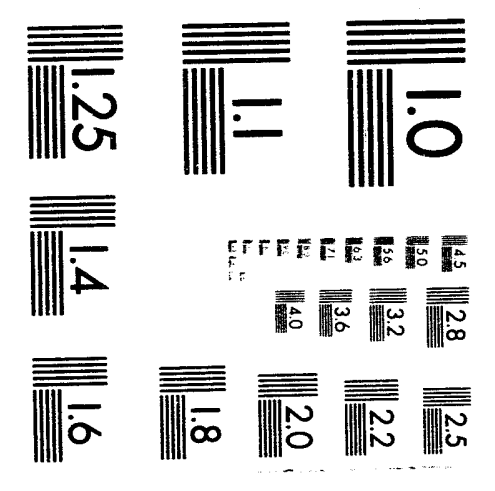



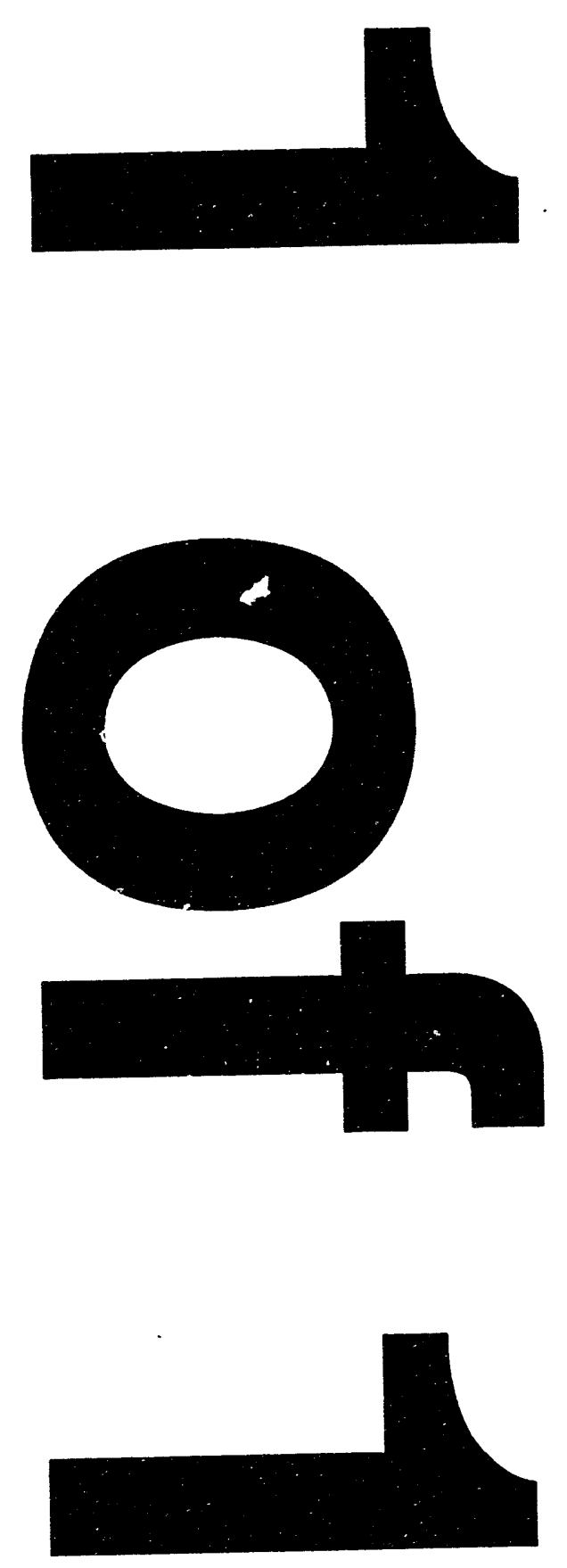
Metals and Ceramics Division

SOFTWARE FOR GONIOMETER CONTROL IN THE TRIPLE ION IMPLANTATION FACILITY

W. R. Allen

Date Published: February 1994

NOTICE: This document contains information of a preliminary nature. It is subject to revision or correction and therefore does not represent a final report.

Prepared for

Office of Basic Energy Sciences

KC 0201040

Prepared by

OAK RIDGE NATIONAL LABORATORY

Oak Ridge, Tennessee 37831-6285

managed by

MARTIN MARIETTA ENERGY SYSTEMS, INC.

for the

U.S. DEPARTMENT OF ENERGY

under contract DE-AC05-84OR21400

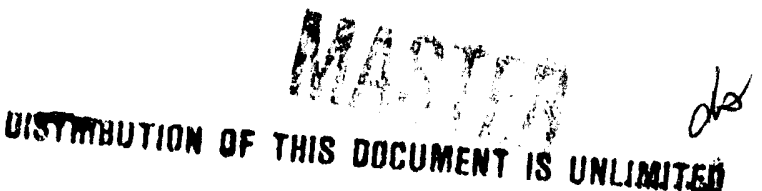


TABLE OF CONTENTS

Page

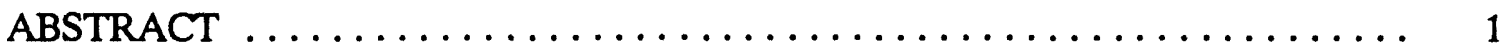

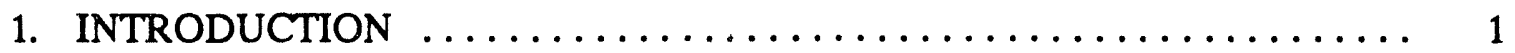

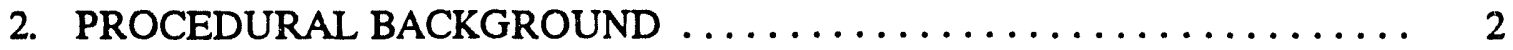

3. SPECIFICS OF THE SOFTWARE $\ldots \ldots \ldots \ldots \ldots \ldots \ldots \ldots \ldots \ldots \ldots \ldots \ldots \ldots \ldots$

4. GUIDELINES FOR OPERATION OF TIF CHANNELING SETUP $\ldots \ldots .5$

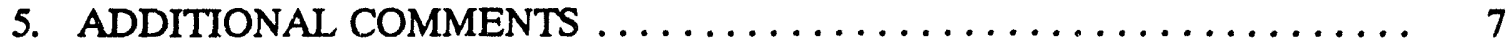

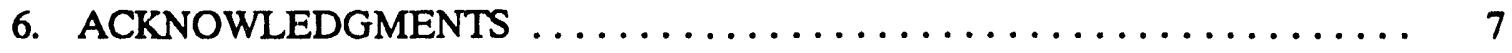

APPENDIX A: COMPUTER PROGRAM FOR GONIOMETER CONTROL ... 9 


\title{
SOFTWARE FOR GONIOMETER CONTROL IN THE TRIPLE ION IMPLANTATION FACILITY ${ }^{*}$
}

\author{
W. R. Allen
}

\begin{abstract}
A computer program is described that controls the goniometer employed in the ion scattering chamber of the Triple Ion Implantation Facility (TIF) in the Metals and Ceramics Division at Oak Ridge National Laboratory. Details of goniometer operation and its incorporation into the ion scattering setup specific to the TIF are also discussed.
\end{abstract}

\section{INTRODUCTION}

Ion-channeling applications in materials science require the accurate positioning of a crystalline direction of high symmetry relative to an analysis ion beam. If the momentum vectors of the incident ion trajectories are nearly collinear with a major crystal axis or plane, the scattering yield from constituent atoms of the host is reduced due to the steering effects of channeling and shadowing. Channeling can be exploited for alignment of a crystal symmetry direction with the ion beam direction. This document presents a computer code written for control of a dual-axis goniometer utilized in the Triple Ion Implantation Facility (TIF) of the Metals and Ceramics Division at the Oak Ridge National Laboratory. A generic goniometer is an instrument that precisely manipulates an attached target with two, or more, orthogonal motion stages. The target is mechanically attached flush to a flat sample holder on the goniometer head, which places it near the intersection of the motion axes. The twoaxis goniometer of the TIF permits a $90^{\circ}$ tilt $(\theta)$ about an axial length of the target and a $360^{\circ}$ rotation $(\phi)$ about an axis perpendicular to the surface normal of the sample holder. This surface normal and the ion beam direction coincide for $\theta=0^{\circ}$. Each axis can be positioned with an accuracy and reproducibility of $0.01^{\circ}$. The muiti-function goniometer

\footnotetext{
"Research supported by the Division of Materials Sciences, U.S. Department of Energy under contract DE-AC05-84OR21400 with Martin Marietta Energy Systems, Inc.
} 
control software facilitates crystal alignment and general target positioning. External device control was required for the stepping motors automating the two motion stages of the goniometer and for two counters interfaced to the data collection electronics.

\section{PROCEDURAL BACKGROUND}

Major crystal planes are identified in the initial channeling alignment phase as the target, tilted several degrees in the azimuthal angle (i.e., $\theta$ ), is rotated in the polar angle (i.e., $\theta$ ) where $\theta=0^{\circ}$ is the ion beam direction. The Miller indices of individual planes are ascertained from relative angular position and the magnitude of the reduction in scattering yield. The coarse angular position of planar intersection should correspond to a major crystal axis. Further rocking in $\theta$ and $\phi$ about this position, while monitoring the scattering yield, can accurately pinpoint the location of a crystal axis relative to the surface normal and to the ion beam direction. Detailed post-alignment measurements along the identified directions of high symmetry can yield information regarding, for example, changes in crystallinity and the lattice location of impurity atoms. A sequence of energy spectra taken about a direction of high symmetry constitutes what is commonly known as an angular scan.

An energy spectrum represents ions scattered from the target during bombardment by an analysis ion beam. These ions can be produced following either elastic or inelastic interactions with target atoms. Biased to create a depletion layer free of charge carriers, a surface-barrier detector positioned in the high-vacuum chamber intercepts all ions incident within a fixed solid angle of acceptance. The number of electron-hole pairs created per ion is proportional to the energy lost in the depletion layer. If the ion is stopped, the number of electron-hole pairs is proportional to the full kinetic energy. A pulse is output to a preamplifier where it is shaped and amplified. A calibrated spectroscopy amplifier further shapes and amplifies the signal. An analog-to-digital converter (ADC) incorporated into a multichannel analyzer (MCA) converts the analog voltage into a digital form. In a process

known as pulse height analysis, the digital signals are sorted according to pulse height to form an energy spectrum. 


\section{SPECIFICS OF THE SOFTWARE}

The computer code for goniometer control was generated with Microsoft QuickBasic version 4.5 for use on IBM-compatible microcomputer (PC). The software code controlling the MCA can be run concurrently. The goniometer control program utilizes a random access memory (RAM) drive, designated as E: drive, to store needed temporary information. A graphics library from SutraSoft, called INGRAF, is applied to graphically present results of alignment diagrams produced by the subroutines PolarGraph and LinearGraph. Descriptions of the routines in the INGRAF library can be found in its documentation. Several custom subroutines, developed by the author, were adapted for use, including those that communicate with the stepping motor controller and provide cursor control on-screen. From within the QuickBasic programming environment, the source code of the goniometer control program can be modified and recompiled into a stand-alone executable form. The INGRAF library must be loaded from the command line with QuickBasic at design time with the sequence "QB/L INGRAF." A complete source listing of the goniometer control program is given in Appendix A.

The PC communicates with a stepping motor controller at a 1200-baud rate with no parity, 7-bit words, and 2 stop bits utilizing RS-232 communications over serial port 1 . The two stepping motors of the goniometer are driven by an indexer/driver commanded by the stepping motor controller. Automated angular scans can be acquired for either $\boldsymbol{\theta}$ or $\phi$. Plots of scattering yield versus angular position are produced graphically, and a hard copy can be output. The screen mode chosen for display is 640 by 480 pixel video graphics array (VGA) graphics, and hard copies are generated on an Epson-compatible printer. Either the video mode or printer type can be modified by altering the appropriate variables in calls from PolarGraph and LinearGraph to the INGRAF subroutine GOPEN.

Operation of the goniometer control software is menu driven with a series of user windows. A menu item is selected with the cursor arrows, or highlighted letter, and the ENTER key pressed to prompt for additional input. Return to the MCA software via "Return to $M C A, "$ not $\mathrm{Ctrl}-\mathrm{C}$ or $\mathrm{Ctrl}-\mathrm{Break}$. These control sequences for program termination are to be utilized if the program hangs or to immediately halt execution of a command. In these instances, the current angular position is irretrievably lost. To recover, the last known position can be input as an offset after returning $\theta$ and $\phi$ to those coordinates using the knobs on the unpowered stepping motors. 
To obviate the slow process of downloading movement parameters at 1200 baud, twelve preset movements for both $\theta$ and $\phi$ are stored in the non-volatile memory of the stepping motor controller. These are accessed from the menu selection "Execute Preset Move." The option "Do Arbitrary Move" permits motion at a preset angular speed for arbitrary values input for $\theta$ and $\phi$. The optimum angular speeds for motion have been determined previously. Home position denotes the values of $\theta$ and $\phi$ which correspond to the ordered pair $\left(0^{\circ}, 0^{\circ}\right)$ as recognized by the code and controller. The menu selection "Home Position Set" assigns the current physical position to $\left(0^{\circ}, 0^{\circ}\right)$. The offset, selected with "Offset Theta/Phi," permits an initial offset relative to the physical position to be entered. Following power up, the controller and code both initialize at $\left(0^{\circ}, 0^{\circ}\right)$. On a shell to the MCA software via "Return to MCA," the angular position is stored on the RAM drive until the goniometer control code is reentered. The menu chr ce "Phi Rock" initiates slow rotation in $\phi$ of the target at $0.25^{\circ} / \mathrm{s}$ while acquiring an energy spectrum having a random-equivalent crystal orientation. The motion proceeds in $\phi$ for the input angular amount in a positive sense, and then the rotation direction is reversed to return to the initial value of $\phi$. Prior to acquisition, an estimate should be made regarding the time required to acquire the randomequivalent energy spectrum. During acquisition, the goniometer control screen should be monitored for an end-of-movement condition. If additional motion is needed, then respond accordingly. The keystrokes Ctrl-C or Ctrl-Break can return the user to the MCA software to view the accumulating energy spectrum. Returning to the goniometer control code presents a blank screen to the user that will refresh only when the rotation ends. Frequently appearing in the body of the code is the instruction "FOR I = 1 TO 100: NEXT I," which inserts a time delay into the code to permit successful communications between the stepping motor controller and the PC.

The PC communicates with two counters via a 32-bit input/output (I/O) card in the back plane of the PC. Using 5-V transistor-transistor logic (TTL), four groups of eight individual bits can be used for input or output. As detailed in the subroutine called Counter, three groups are used here for receiving the hexadecimal number of counts indicated on the display of one counter. This counter has a peripheral interface that permits the above operations to be performed. Pin 47 monitors the input from the interval BNC connection on the counter, which indicates a high logic level when a preset is achieved. One group outputs a TTL high signal to reset and zero both counters. 
As mentioned previously, manual and automatic modes of angular scanning for crystal alignment are possible. If the automated angular scan mode is desired, connect the ribbon cable between the digital $\mathrm{I} / \mathrm{O}$ card interface in the back plane of the control computer and the interface of a counter. This counter registers the accumulated number of scattering events in an energy (i.e., pulse height) range specified by the output of a single channel analyzer (SCA). For manual operation, disconnect this cable. A second counter monitors the output of a current digitizer sensing the beam current on target. An energy spectrum is acquired for a specific fluence of analysis ions. When the preset is achieved, a trigger signal from the counter halts counting by the MCA. In addition, the ion beam is automatically shuttered by inserting a Faraday cup to minimize unwanted damage to the target. The number of scattering events in the range specified by the SCA window is recorded and the target indexed to the next orientation. In automatic mode, the $\mathrm{PC}$ positions the target in $\theta$ and $\phi$ according to input parameters, reads and records the number of counts, and resets both counters. In manual mode, the number of counts is recorded by hand, the counters reset manually with a remote switch, and the target repositioned as necessary by a series of movements.

To acquire an energy spectrum, the angular position of the sample is set, and one returns to the MCA control code. An energy spectrum is acquired for a preset dose of analysis ions chosen with the preset on the counter interfaced to the current integrator. The energy spectrum is stored, and one shells to the goniometer control code for repositioning of the sample. This process is repeated as necessary.

\section{GUIDELINES FOR OPERATION OF THE TIF CHANNELING SETUP}

Power up the microcomputer located on a table near the south wall in the console room. To execute the software for the MCA, type MCA.BAT(RET). At runtime, the batch file MCA.BAT provides command-line switches to the MCA control software. Two MCA cards, manufactured by The Nucleus, are present in the back plane of the PC.

Energize the two stepping motors of the goniometer via the stepping motor controller that is positioned on a shelf in the southeast corner of the shielded target room adjacent to the scattering chamber. The key switch should be positioned in the REMOTE position. Prior to turning the ac power for the controller on, ensure that the electrical connections are 
closed between the motors and power supply and the controller and power supply, respectively. Also, check that the RS-232 cable is connected. Enable RS-232 communication with a baud rate of 1200 bits per second (bps) using the proper sequence of keystrokes from the keypad on the front face of the stepping motor controller. The stepping motors should be energized and available for computer control at this stage.

From the "EXECUTE A BATCH FILE COMMAND" on the pull-down menu within the MCA program, execute "C:path $\backslash$ GON.EXE" to switch the focus to the goniometer control program. Initiation of the goniometer control program will occur if a communications link is established with the stepping motor controller. Data acquisition launched prior to shelling to the goniometer control program proceeds uninhibited.

The ion beam is aligned and collimated with the assistance of a thin, flat $\mathrm{Al}_{2} \mathrm{O}_{3}$ (or quartz) specimen mounted at the target position on the goniometer head. The beam spot glows bright blue. Place the beam initially on target by steering with magnetic field adjustments and X-Y steerer variations at the console and by vertical adjustment of the height of the goniometer head. Adjustment of vertical and horizontal pairs of slits (i.e., the "jaws") in the magnet room is facilitated by monitoring target current with the current integrator in a nuclear instrument module (NIM) rack adjacent to the target chamber. A remote meter near the jaws assembly permits beam current to be observed during adjustment. With the beam on target, insert the large aperture on the linear translation feedthrough. Minor adjustment of the scattering chamber position relative to the beam line can place the beam on target center. The beam position on target can be alternatively adjusted with coordinated magnetic steering and movement of jaw pairs. Insert the fixed aperture on the rotary feedthrough, choosing the central hole. The fixed aperture has three openings of varying size that can be selected by a minor rotation. Further adjustment of the individual jaws is now possible for finer collimation of the beam spot and further reduction of the angular divergence. A gold, thin film is present on the mask that mechanically attaches the target to the goniometer head. Backscattered ions from it appear distinctively in the energy spectrum and serve as an aid in positioning.

Secondary electron emission from the target during analysis ion bombardment is suppressed by a $50-\mathrm{V}$ dc battery located on a shelf in the instrument rack in the console room. To extend battery life, disconnect the BNC cable that links the digital current integrator and the physical target during extended idle periods. 
From within the MCA shell, an energy spectrum is briefly acquired to indicate the composition of the sample. An energy window can be set on the energy spectrum utilizing an SCA and a calibrated pulser. A range of pulse heights from the spectroscopy amplifier is passed to an external counter and rate meter. Preferably, this range corresponds to scattering from a single bulk element of significant abundance in the sample. Scattering within this range is also from a depth region expected to be crystalline. For alignment purposes, the number of scattering events per unit analysis ion dose (i.e., accumulated charge) collected in this region of interest is observed as a function of angular position. A typical preset ion dose is of the order of $0.1 \mathrm{nC}$.

\section{ADDITIONAL COMMENTS}

Instructions for movements of each stepping motor are contained in specific subroutines in the non-volatile memory of the stepping motor controller. Movement distance, sense, and speed can be altered from the keypad of the stepping motor controller or via serial communication. The program section labeled ArbMove can be modified to stand alone for use in modifying individual subroutines.

Embellishments could be made to the code to enhance its usefulness in the TIF. Since the control source code for the MCA written in $\mathrm{C}$ is provided by the MCA vendor (i.e., The Nucleus in Oak Ridge, Tennessee), the goniometer control program could be interfaced to automatically generate full angular scans. The procedure would entail positioning the crystal with the goniometer, shelling to a program to control the MCA, counting and electronically recording an energy spectrum, returning to the goniometer control code, repositioning the target, and repeating the process. User judgment would be required to properly adjust the angular position of the target between consecutive energy spectra. Another direction for growth would be to port the application to VisualBasic to create an enhanced user interface.

\section{ACKNOWLEDGMENTS}

The author would like to thank J. D. Hunn, M. B. Lewis, and L. K. Mansur for their assistance in reviewing this manuscript; M. W. Terrell for secretarial services; G. R. Carter for manuscript preparation; and $\mathrm{K}$. Spence for editing. 


\section{APPENDIX A}

\section{COMPUTER PROGRAM FOR GONIOMETER CONTROL}

' \$INCLUDE: 'C:IQB45IQBFILESIG.BI

'The included file can be found at the end of this source listing.

DIM w1 AS WindowsType, w5 AS WindowsType, w6 AS WindowsType, w7 AS WindowsType DIM w1Text\$(1 TO 9), w5Text\$(1 TO 2), w6Text\$(1 TO 13), w7Text\$(1 TO 2)

$\operatorname{DIM} \mathrm{X}(25), \mathrm{Y}(25)$

DIM MoveAngle(25), MasterTxtTheta\$(13), MasterTxtPhi\$(13)

OPEN "COM1:1200,N,7,2,RS,CS,DS,CD,LF" FOR RANDOM AS \# 1

' Retrieve the file containing previous theta and phi offsets.

'These are retained on normal program termination for use upon reentry.

OPEN "E:IGONIOM.PAR" FOR RANDOM AS \#4

GET \#4, 1, OffTheta

GET \#4, 2, OffPhi

CLOSE \#4

' List of menu items for motion.

'The corresponding movements are stored in the non-volatile memory of the controller.

MasterTxtTheta $\$(1)=$ " +0.05 deg."

MasterTxtTheta $\$(2)=$ "+0.10 deg."

MasterTxtTheta $\$(3)=$ "+0.20 deg."

MasterTxtTheta $\$(4)=$ "+0.50 deg."

MasterTxtTheta\$(5) = "+1.00 deg."

MasterTxtTheta $\$(6)=$ "+5.00 deg."

MasterTxtTheta $\$(7)=$ "-0.05 deg."

MasterTxtTheta $\$(8)=$ "-0.10 deg."

MasterTxtTheta $\$(9)=$ "-0.20 deg."

MasterTxtTheta $\$(10)=$ "-0.50 deg."

MasterTxtTheta $\$(11)=$ "-1.00 deg."

MasterTxtTheta\$(12) = "-5.00 deg."

MasterTxtTheta $\$(13)=$ "Change to Phi"

MasterTxtPhi $\$(1)=$ " $+0.50 \mathrm{deg} . "$

MasterTxtPhi $\$(2)=$ " +1.00 deg."

MasterTxtPhi $\$(3)=$ " +2.00 deg."

MasterTxtPhi $\$(4)=$ " +5.00 deg."

MasterTxtPhi $\$(5)=$ "+10.00 deg."

MasterTxtPhi $\$(6)=$ "+20.00 deg."

MasterTxtPhi\$(7) = " $-0.50 \mathrm{deg} . "$

MasterTxtPhi\$(8) = " -1.00 deg."

MasterTxtPhi\$(9) = " -2.00 deg."

MasterTxtPhi $\$(10)=$ " -5.00 deg."

MasterTxtPhi $\$(11)=$ "-10.00 deg."

MasterTxtPhi\$(12) = "-20.00 deg."

MasterTxtPhi\$(13) = "Change to Theta"

'The following sets of statements set parameters for screen creation.

w5. action $=-1$

w5.bgdEdge $=$ Green: $w 5$. fgdEdge $=$ LightBlue

w5.bgdBody $=$ Green: $w 5$. fgdBody $=$ Black 
w5.fgdTitle $=$ Green: $w 5$. bgdTitle $=$ LightBlue w5.fgdPrompt $=0$ : $w 5$. bgdPrompt $=0$

w5. fgdHilite $=0:$ w5.bgdHilite $=0$

w5.fgdBorder $=$ Green: w5.bgdBorder $=$ Blue

w5.screenColor = Cyan

w5Title $\$=$ "Position"

w5Prompt\$ = "'"

w5.displayPage $=0: w 5$. createPage $=2:$ w 5 .overPage $=-1$

w5.row $=17$ : $w 5$.column $=37$

w1Prompt $\$=" '$

w1Title $\$=$ "GONIOMETER CONTROL"

w1Text $\$(1)=$ "Auto-Alignment"

w1Text\$(2) = "Do Arbitrary Move"

w1Text $\$(3)=$ "Execute Preset Move"

w1Text $\$(4)=$ "Home Position Set"

w1Text $\$(5)=$ "Offset Theta/Phi"

w1Text $\$(6)=$ "Phi Rock"

w1Text\$(7) = "Quit to DOS"

w1Text\$(8) = "Return to MCA"

w1Text $(9)=$ "Scan"

$w 1$ displayPage $=0: w 1$.createPage $=1: w 1$.overPage $=2$

w1.row $=4:$ w1 column $=5$

w1.action $=1$

w1.fgdEdge $=$ LightMagenta: $w 1$.bgdEdge $=$ Blue

w1.fgdBody $=$ White: $w 1$. bgdBody = Blue

w1.fgdTitle = LightRed: w1.bgdTitle = Blue

w1.fgdPrompt $=$ LightGreen: $w 1$.bgdPrompt $=$ Blue

w1.fgdHilite = BrightWhite: $w 1$.bgdHilite = LightCyan

w1.fgdBorder $=-1$ : w1.bgdBorder $=0$

w1.screenColor $=-1$

w6.action $=1$

w6.fgdEdge = LightMagenta: w6.bgdEdge = Blue

w6.fgdBody = White: $w 6$. bgdBody = Blue

w6.fgdTitle = LightRed: w6.bgdTitle = Blue

w6.fgdPrompt = LightGreen: w6.bgdPrompt = Blue

w6.fgdHilite $=$ BrightWhite: $w 6$. bgdHilite = LightCyan

w6.fgdBorder $=$ LightBlue: w6.bgdBorder $=$ Blue

w6.screenColor $=$ Cyan

w6Prompt\$ = "'

$w 7$. action $=-1$

w7.bgdEdge $=$ Green: $w 7$. fgdEdge $=$ LightBlue

$w 7$. bgdBody $=$ Green: $w 7$. fgdBody $=$ Black

w7.fgdTitle $=$ Green: $w 7$. bgdTitle $=$ LightBlue

w7.fgdPrompt $=0$ : $w 7$.bgdPrompt $=0$

$w 7$. fgdHilite $=0:$ 7. bgdHilite $=0$

w7.fgdBorder $=$ Green: $w 7$. bgdBorder $=$ Blue

w7.screenColor $=$ Cyan

wTTitle\$ = "'"

w7Prompt $\$=" '$

$w 7$.displayPage $=7: w 7$. createPage $=2: w 7$. overPage $=-1$

$w 7$ row $=10: w 7$.column $=47$ 


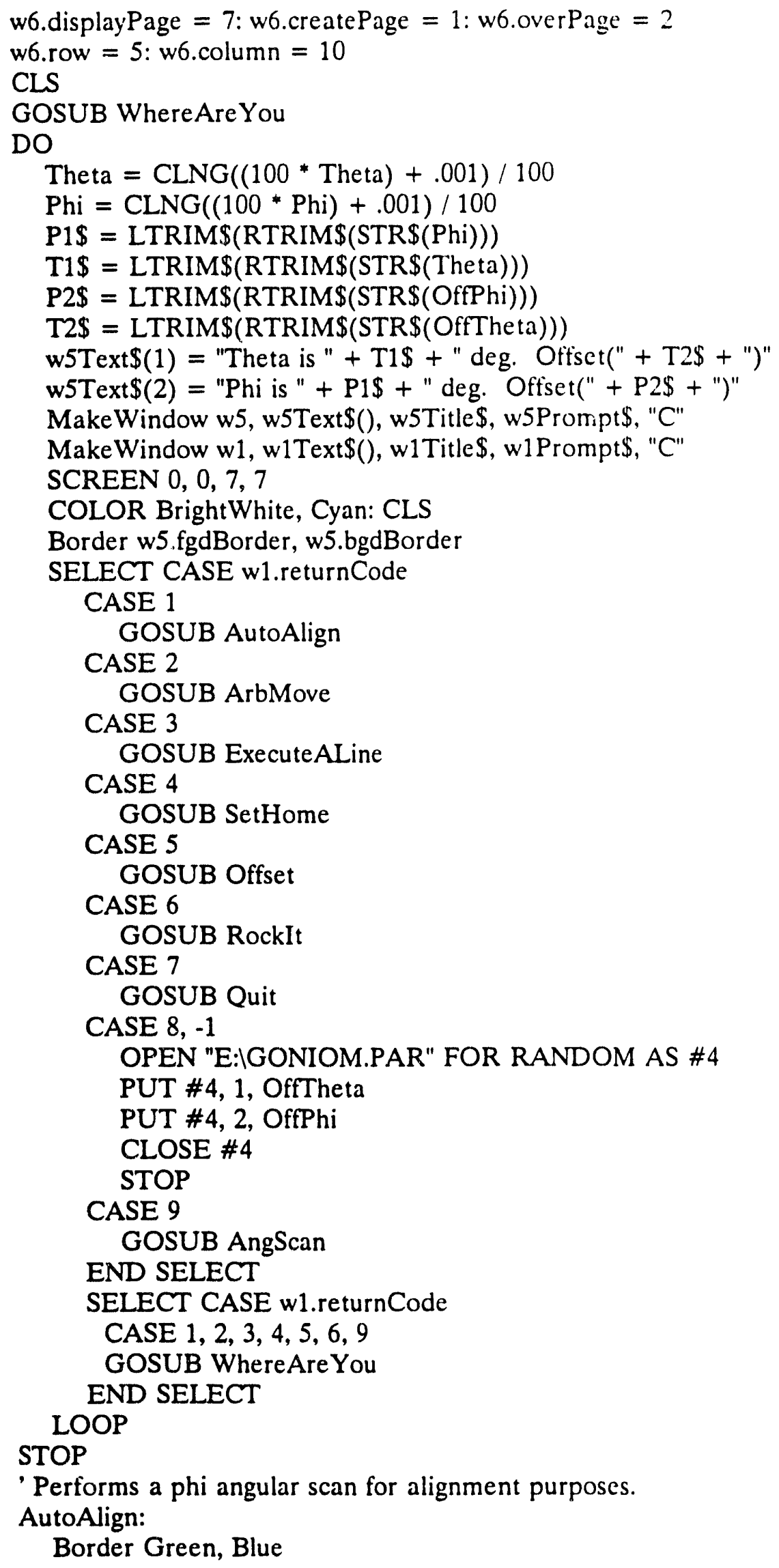


COLOR Black, Green

LOCATE 4, 18: PRINT SPACE\$(41)

LOCATE 6, 18: PRINT SPACE \$(41)

LOCATE 5, 18

PRINT " Automatic Crystal Alignment Procedure "

COLOR BrightWhite, Blue

DO:

LOCATE 8,8

INPUT " Choose the angular interval to scan in degrees (0-359) "; AngInt

IF AngInt $=0$ THEN RETURN

LOOP UNTIL AngInt $>0$ AND AngInt $<360$

DO:

LOCATE 10,8

INPUT " Choose the scan Direction (+ or -) "; AngDir\$

LOOP UNTIL AngDir\$ = "+" OR AngDir\$ = "."

DO:

LOCATE 12,8

PRINT " Choose the angular increment $(0.5$ or 1.0$)$ ";

INPUT AngInc

LOOP UNTIL AngInc $=.5$ OR AngInc $=1 !$

LOCATE 14, 25: COLOR BrightWhite, Red

INPUT " Continue (Y/N) "; GoOn\$

IF LEFT\$(UCASE\$(GoOn\$), 1) $<>$ "Y" THEN RETURN

IF AngDir\$ = "+" THEN

IF AngInc $=.5$ THEN

SubNum $=13$

ELSEIF AngInc $=1$ ! THEN

SubNum $=14$

END IF

ELSEIF AngDir\$ = "-" THEN

IF AngInc $=.5$ THEN

SubNum $=19$

ELSEIF AngInc $=1$ ! THEN

SubNum $=20$

END IF

END IF

JMax = INT(AngInt / AngInc)

DummyPhi $=$ Phi

DummyTheta $=$ Theta

SumTotal $=0$

COLOR White, Blue

CLS

Border Green, Blue

LOCATE 6, 24

COLOR 31, Green

PRINT " ALIGNMENT IN PROGRESS "

COLOR BrightWhite, Blue

LPRINT "Theta", "Phi", "Counts"

REDIM Counts(400), PhiVal(400), NrmYld(400), Plane(30), PlaneYld(30)

FOR $\mathrm{J}=0$ TO JMax

IF $\mathrm{J} \ll 0$ THEN 


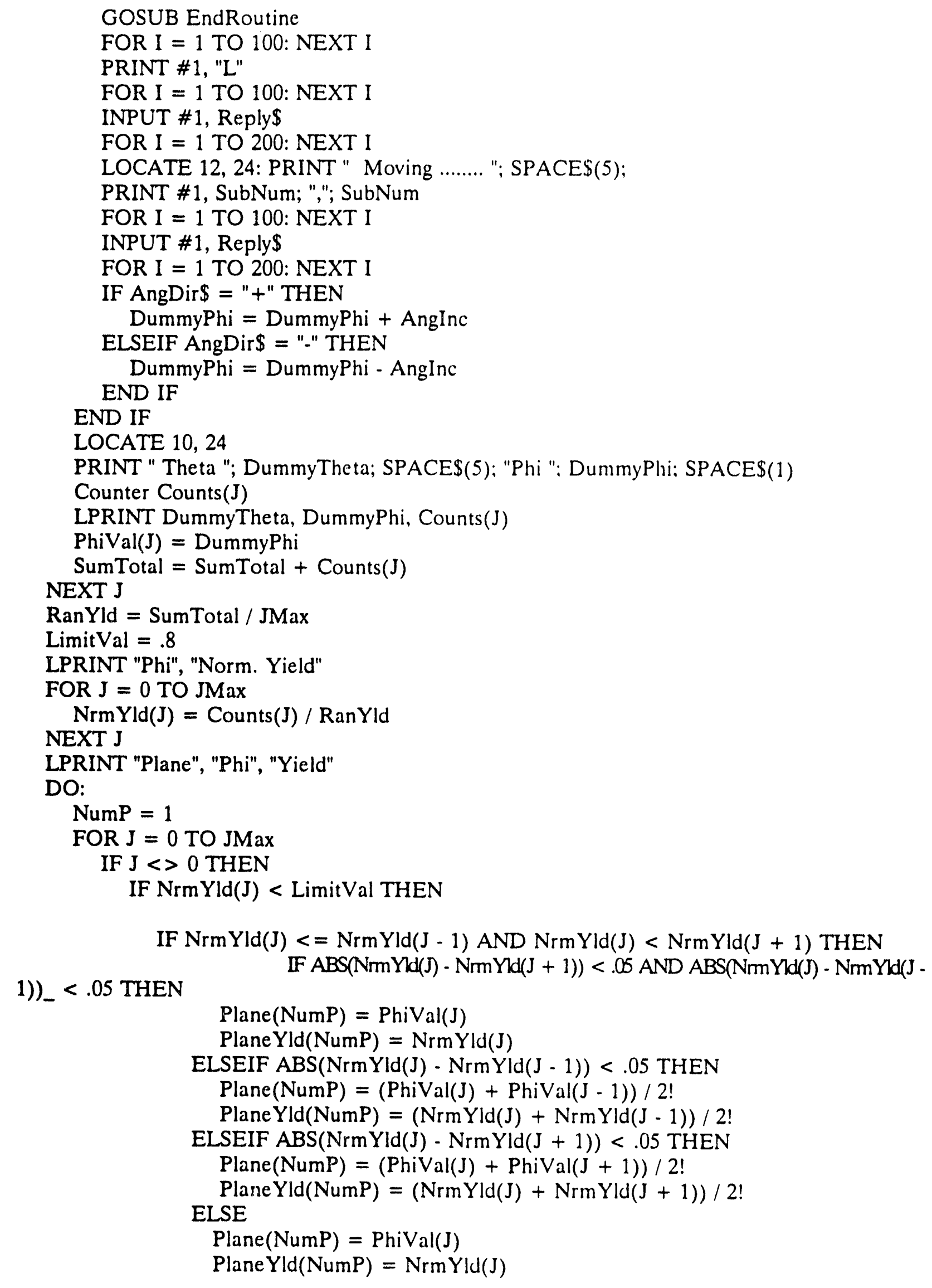




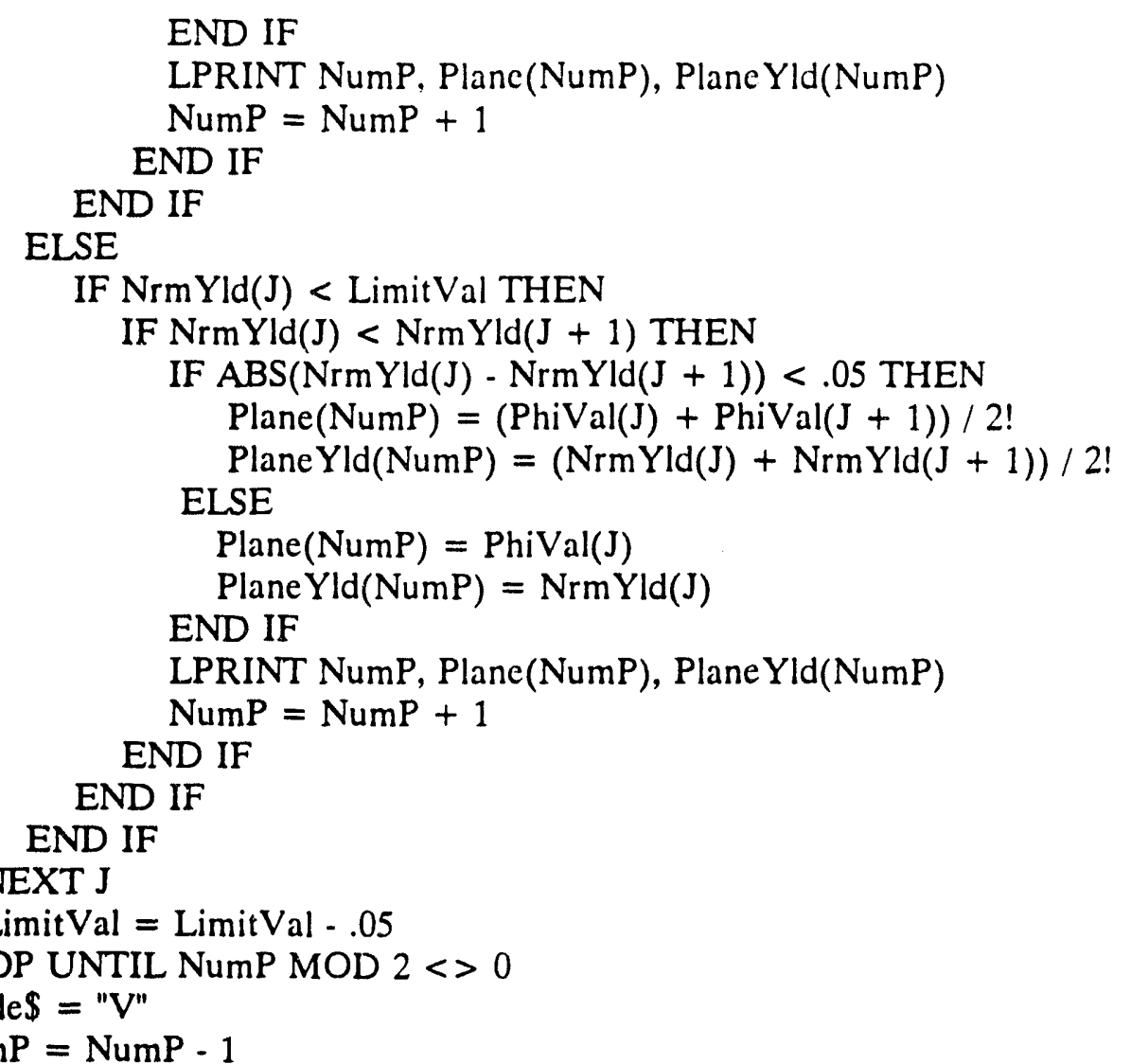

END IF

LPRINT NumP, Planc(NumP), Plane Yld(NumP)

END IF

END IF

IF NrmYld(J) < LimitVal THEN

IF NrmYld $(J)<\operatorname{Nrm} Y l d(J+1)$ THEN

Plane(NumP) $=$ (PhiVal(J) + PhiVal $(J+1)) / 21$

PlaneYld(NumP $)=(\operatorname{NrmYld}(J)+\operatorname{NrmYld}(J+1)) / 2$ !

Plane $($ NumP $)=$ PhiVal $(\mathrm{J})$

Plane Yld $($ NumP $)=$ NrmYld $(J)$

LPRINT NumP, Plane(NumP), Plane Yld(NumP)

END IF

END IF

LimitVal $=$ LimitVal -.05

LOOP UNTIL NumP MOD $2<>0$

NEXT J

Mode $\$=$ "V"

NumP $=$ NumP -1

CALL PolarGraph(Mode\$, NumP, DummyTheta, Plane(), Plane Yld())

SCREEN 0, 0, 0, 0

COLOR BrightWhite, Blue

CLS

LOCATE 10, 15

INPUT "Do you want a hardcopy of the alignment diagram"; HardOn\$

IF LEFT\$(UCASE\$(HardOn\$), 1) = "Y" THEN

CALL PolarGraph("E", NumP, DummyTheta, Plane(), Plane Yld())

END IF

SCREEN 0, 0, 0, 0

COLOR BrightWhite, Blue

CLS

ERASE Counts, PhiVal, NrmYld, Plane, Plane Yld

RETURN

- Performs an automated angular scan in either theta or phi.

AngScan:

Border Green, Blue

COLOR Black, Green

LOCATE 4, 18: PRINT SPACE\$(38)

LOCATE 6, 18: PRINT SPACE\$(38)

LOCATE 5,18

PRINT " Automatic Angular Scan Procedure "

COLOR BrightWhite, Blue

LOCATE 8,8

INPUT " Choose motion - Theta(T) or Phi(P)"; Motor\$

Motor $\$=$ LEFT\$(UCASE\$(Motor\$), 1) 
DO:

LOCATE 10, 8

INPUT " Choose the angular interval to scan in degrees (0-360) ": AngInt

IF AngInt $=0$ THEN RETURN

LOOP UNTIL AngInt $>0$ AND AngInt $<361$

DO:

LOCATE 12,8

INPUT " Choose the scan Direction (+ or -) "; AngDir\$

LOOP UNTIL AngDir\$ = "+" OR AngDir\$ = "-"

DO:

LOCATE 14,8

IF Motor $\$=$ "T" THEN

PRINT " Choose the angular increment $(0.1,0.2$. or 0.5$)$ "

ELSE

PRINT " Choose the angular increment $(0.5,1.0 .2 .0$ or 5.0$) "$

END IF

LOCATE 16, 20

INPUT " Increment "; AngInc

LOOP UNTIL AngInc $=.1$ OR AngInc $=.2$ OR AngInc $=.5$ OR AngInc $=1$ OR AngInc $=$

2!_ OR AngInc $=5$ !

LOCATE 18, 8: COLOR LightGreen, Blue

INPUT " Continue (Y)"; GoOn\$

COLOR White, Reú

IF LEFT\$(UCASE\$(GoOn\$), 1) $<$ "Y" THEN RETURN

IF Motor $\$=$ "T" THEN

IF AngDir\$ = "+" THEN

IF AngInc $=.1$ THEN

SubNum $=2$

ELSEIF AngInc $=.2$ THEN

SubNum $=3$

ELSEIF AngInc $=.5$ THEN

SubNum $=4$

END IF

ELSEIF AngDir\$ = "-" THEN

IF AngInc $=.1$ THEN

SubNum $=8$

ELSEIF AngInc $=.2$ THEN

SubNum $=9$

ELSEIF AngInc $=.5$ THEN

SubNum $=10$

END IF

END IF

ELSEIF Motor\$ = "P" THEN

IF AngDir\$ = "+" THEN

IF AngInc $=.5$ THEN

SubNum $=13$

ELSEIF AngInc = 1! THEN

SubNum $=14$

ELSEIF AngInc = 2! THEN

SubNum $=15$

ELSEIF AngInc = 5! THEN 


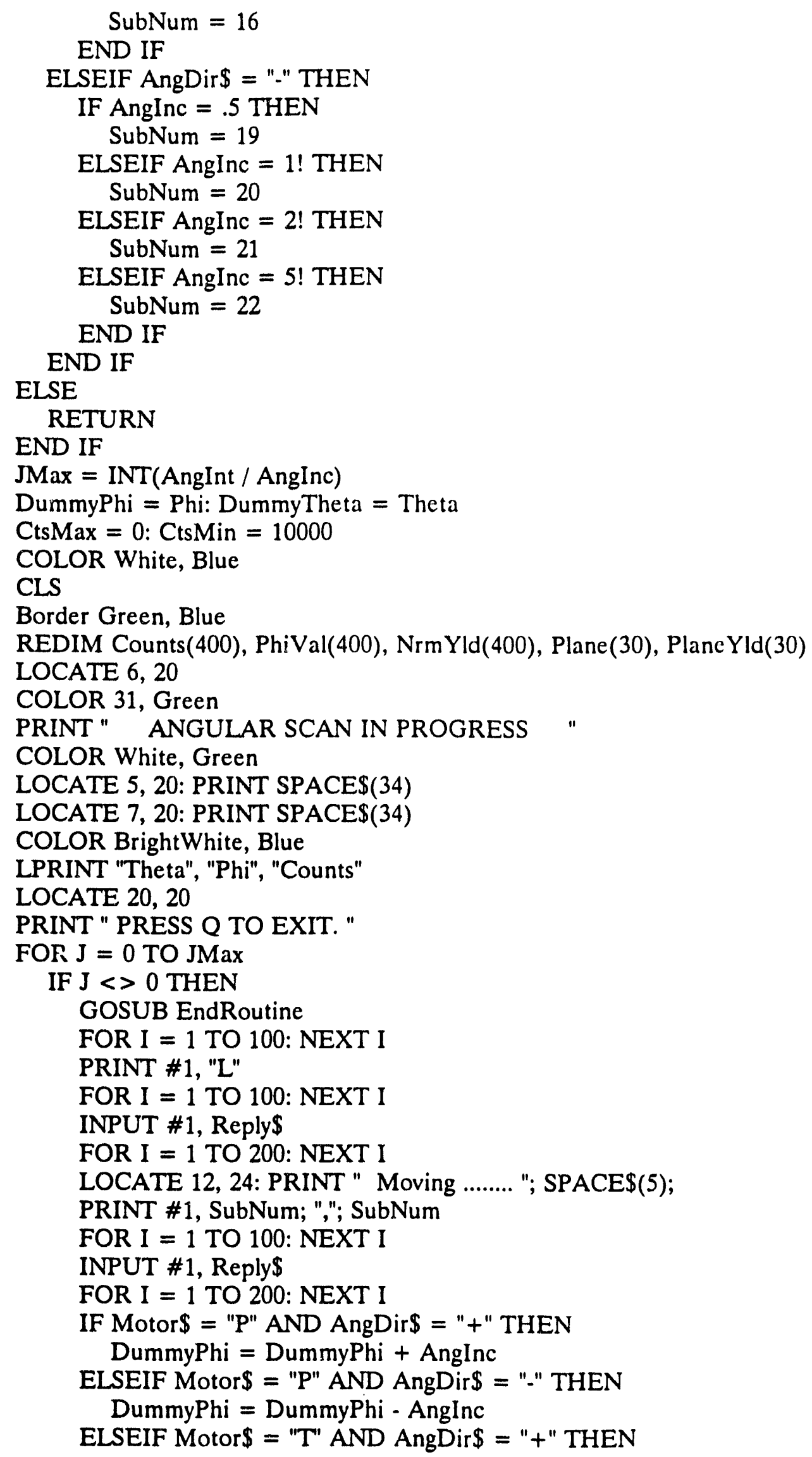




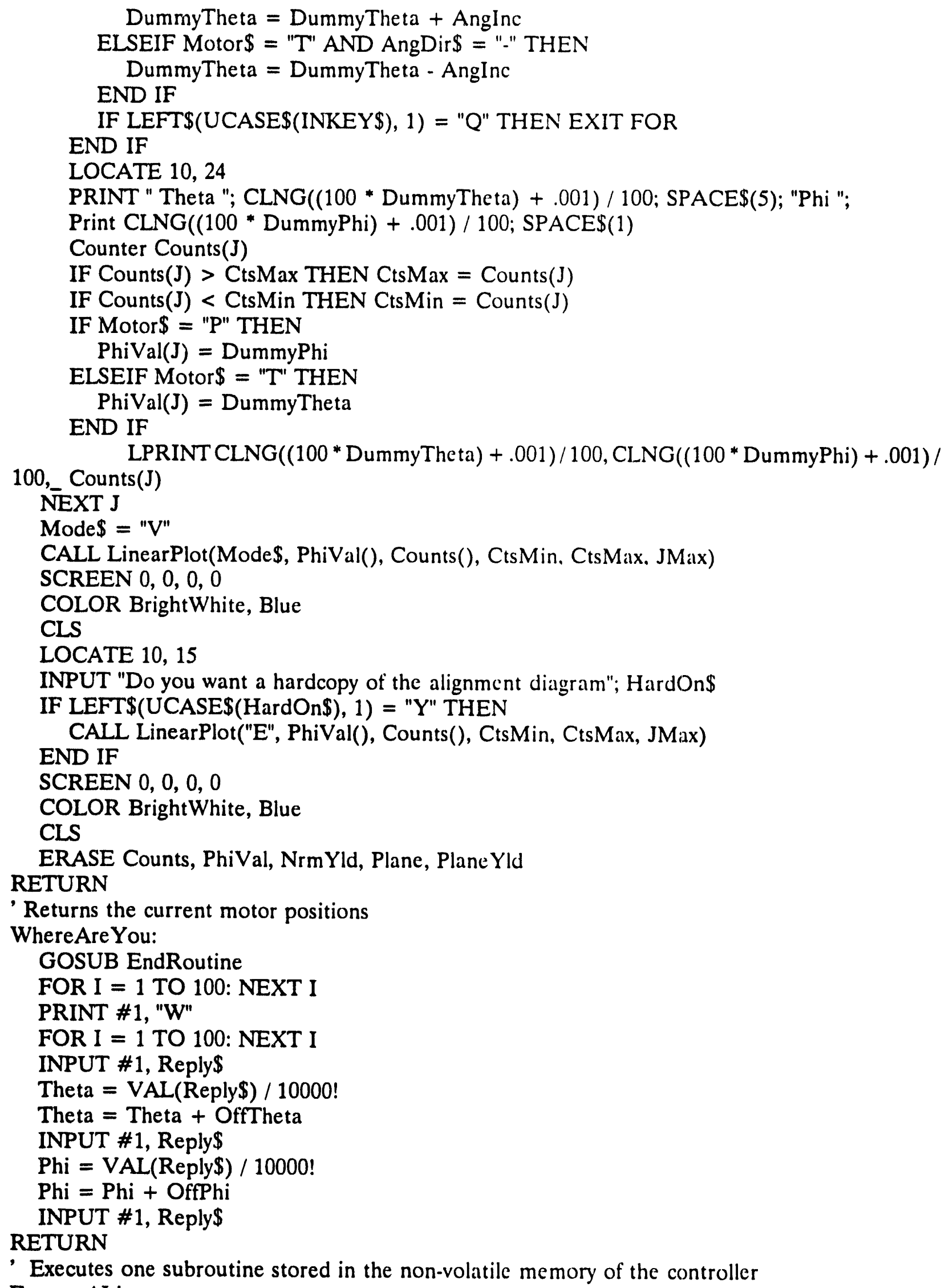

- Executes one subroutine stored in the non-volatile memory of the controller ExecuteAline: 
DummyPhi $=$ Phi

DummyTheta $=$ Theta

LOCATE 12, 35

PRINT " Please Wait "

GOSUB EndRoutine

FOR I = 1 TO 100: NEXT I

PRINT \#1, "L"

FOR I = 1 TO 100: NEXT I

INPUT \#1, Reply\$

FOR I = 1 TO 100: NEXT I

InitMotor\$ = "T"

LOCATE 12, 35

PRINT SPACE\$(13)

DO:

DO:

DummyTheta $=\operatorname{CLNG}((100 *$ DummyTheta $)+.001) / 100$

DummyPhi $=$ CLNG $\left(\left(100^{*}\right.\right.$ DummyPhi $\left.)+.001\right) / 100$

wTText\$(1) = "Theta " + LTRIM\$(RTRIM\$(STR\$(DummyTheta))) + " deg."

wTText\$(2) = "Phi_" + LTRIM\$(RTRIM\$(STR\$(DummyPhi))) + " deg."

MakeWindow w7, w7Text\$(), w7Title\$, w7Prompt\$, "'

FOR I = 1 TO 100: NEXT I

IF InitMotor\$ = "T" THEN

FOR Kntr $=1$ TO 13

w6Text $\$(K n t r)=$ MasterTxtTheta\$(Kntr)

NEXT Kntr

w6Title\$ = "Theta Position"

ELSEIF InitMotor\$ = "P" THEN

FOR Kntr $=1$ TO 13

w6Text\$(Kntr) = MasterTxtPhi\$(Kntr)

NEXT Kntr

w6Title $\$=$ "Phi Position"

ELSE

RETURN

END IF

MakeWindow w6, w6Text\$(), w6Title\$, w6Prompt\$, "'

IF InitMotor $\$=$ "P" THEN

OffSub $=12$

ELSE

OffSub $=0$

END IF

SELECT CASE w6.returnCode

CASE 1

SubNum1 $=1+$ OffSub

IF InitMotor $\$=$ "T" THEN DummyTheta $=$ DummyTheta +.05

IF InitMotor $\$=$ "P" THEN DummyPhi $=$ DummyPhi +.5

CASE 2

SubNum1 $=2+$ OffSub

IF InitMotor $\$=$ "T" THEN DummyTheta $=$ DummyTheta +.1

IF InitMotor\$ = "P" THEN DummyPhi $=$ DummyPhi +1 !

CASE 3

SubNum $1=3+$ OffSub 
IF InitMotor $\$=" T$ " THEN DummyTheta $=$ DummyTheta +.2

IF InitMotor $\$=$ "P" THEN DummyPhi $=$ DummyPhi +2 !

CASE 4

SubNum1 $=4+$ OffSub

IF InitMotor $\$=$ "T" THEN DummyTheta $=$ DummyTheta +.5

IF InitMotor $\$=$ "P" THEN DummyPhi = DummyPhi +5 !

CASE 5

SubNum1 $=5+$ OffSub

IF InitMotor $\$=$ "T" THEN DummyTheta = DummyTheta +1 !

IF InitMotor $\$=$ "P" THEN DumnyPhi = DummyPhi +10 !

CASE 6

SubNum1 $=6+$ OffSub

IF InitMotor $\$=$ "T" THEN DummyTheta $=$ DummyTheta +5 !

IF InitMotor $\$=$ "P" THEN DummyPhi = DummyPhi +20 !

CASE 7

SubNum $1=7+$ OffSub

IF InitMotor $\$=$ "T" THEN DummyTheta $=$ DummyTheta -.05

IF InitMotor $\$=$ "P" THEN DummyPhi $=$ DummyPhi -.5

CASE 8

SubNum $1=8+$ OffSub

IF InitMotor $\$=$ "T" THEN DummyTheta $=$ DummyTheta -.1

IF InitMotor $\$=$ "P" THEN DummyPhi = DummyPhi -1 !

CASE 9

SubNum1 $=9+$ OffSub

IF InitMotor $\$=$ "T" THEN DummyTheta $=$ DummyTheta -.2

IF InitMotor $\$=$ "P" THEN DummyPhi = DummyPhi -2 !

CASE 10

SubNum1 $=10+$ OffSub

IF InitMotor $\$=$ "T" THEN DummyTheta $=$ DummyTheta -.5

IF InitMotor $\$=$ "P" THEN DummyPhi = DummyPhi -5 !

CASE 11

SubNum1 $=11+$ OffSub

IF InitMotor\$ = "T" THEN DummyTheta = DummyTheta -1 !

IF InitMotor $\$=$ "P" THEN DummyPhi = DummyPhi -10 !

CASE 12

SubNum1 $=12+$ OffSub

IF InitMotor\$ = "T" THEN DummyTheta = DummyTheta -5 !

IF InitMotor\$ = "P" THEN DummyPhi = DummyPhi -20 !

CASE 13

IF InitMotor $\$=$ "P" THEN

InitMotor $\$=$ " $\mathrm{T}$ "

ELSEIF InitMotor $\$=$ "T" THEN

InitMotor\$ = "P"

END IF

EXIT DO

CASE -1

RETURN

END SELECT

LOCATE 22, 50

COLOR BrightWhite, Red

PRINT " WORKING ..... " 


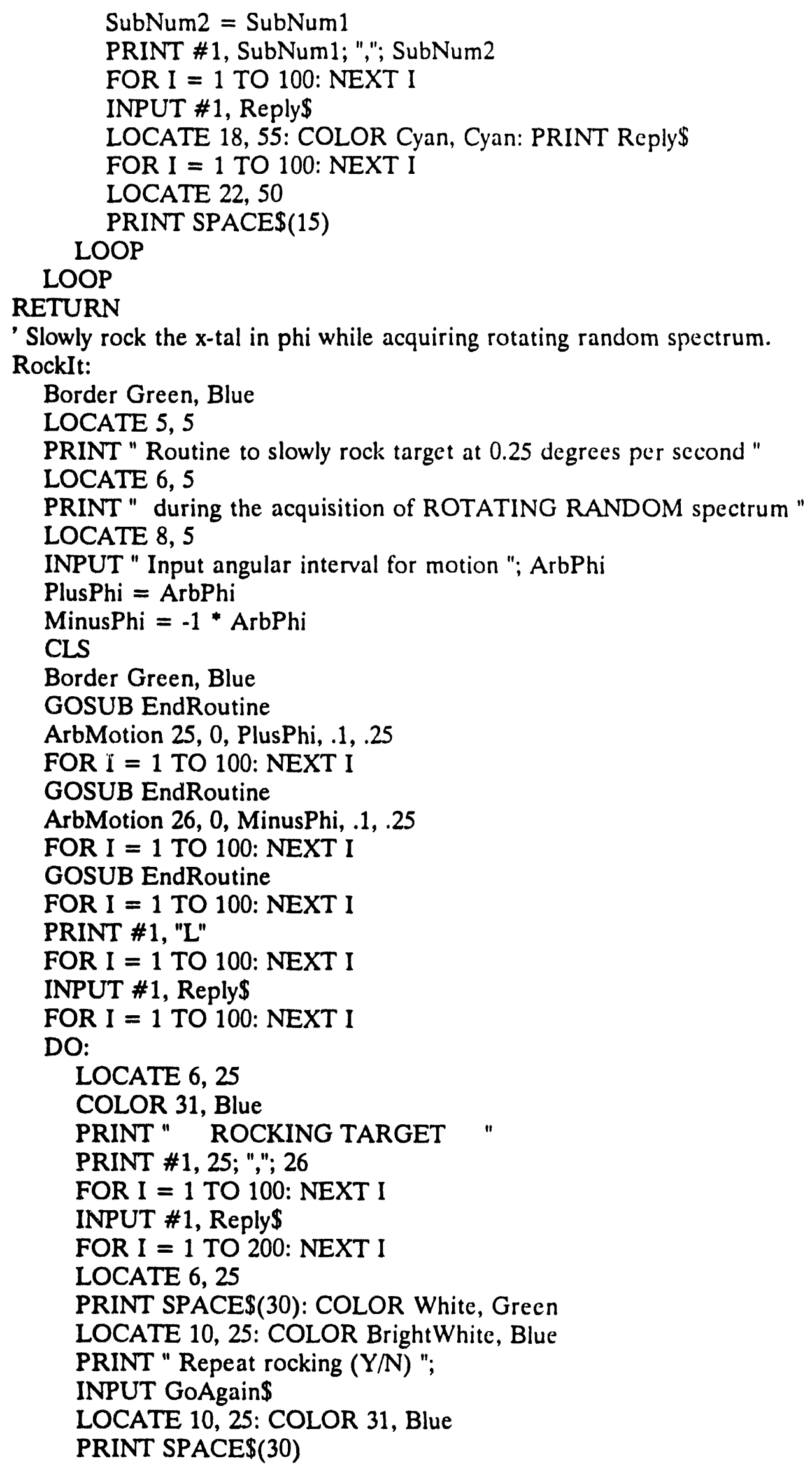




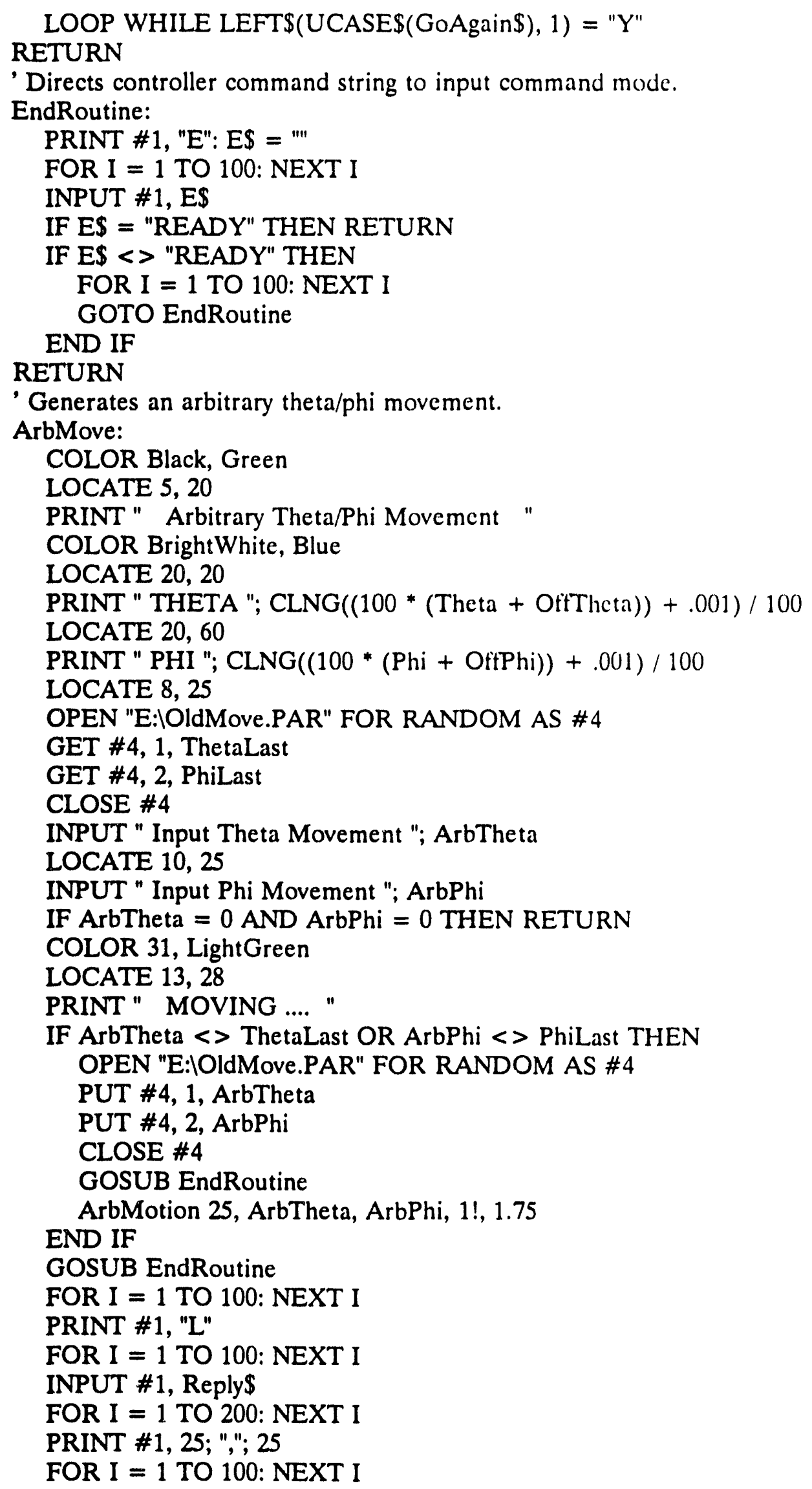


INPUT \#1, Reply\$

FOR I = 1 TO 200: NEXT I

RETURN

' Sets the home position (i.e., theta and phi=0).

SetHome:

GOSUB EndRoutine

LOCATE 12, 25: COLOR BrightWhite, Bluc

INPUT " Confirm Set Home (N)"; Confirm\$

IF LEFT\$(UCASE\$(Confirm\$), 1) $<>$ "Y" THEN RETURN

FOR I = 1 TO 100: NEXT I

PRINT \# 1 , "S"

FOR I = 1 TO 100: NEXT I

INPUT \# 1 , S\$

FOR I = 1 TO 200: NEXT I

RETURN

- An offset can occur since the controller considers theta and phi to

'be zero on power up. This routine permits adjustment for this.

Offset:

LOCATE 10, 20: COLOR BrightWhite, Bluc

INPUT " THETA offset in degrees"; OffTheta

LOCATE 12, 20

INPUT " PHI offset in degrees"; OffPhi

RETURN

'Exit program routine.

Quit:

GOSUB EndRoutine

INPUT "Confirm Exit (N)"; Confirm\$

IF LEFT\$(UCASE\$(Confirm\$), 1) $<>$ "Y" THEN RETURN

PRINT \#1, "Q"

CLOSE \#1

END

STOP

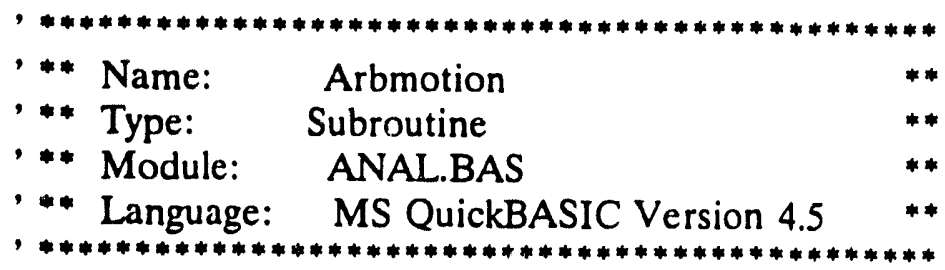

- Permits an arbitrary movement in $\theta$ and/or $\phi$

' EXAMPLE OF USE: ArbMotion (SubNum\%, TAmount, PAmount, TSpeed, PSpeed)

'PARAMETERS:

' MODULE LEVEL

TAmount Theta movement

PAmount Phi movement

TSpeed Theta motor speed

PSpeed Phi motor speed

' DECLARATIONS: DECLARE SUB ArbMotion (SubNum\%, TAmount, PAmount, TSpeed, PSpeed)

SUB ArbMotion (SubNum\%, TAmount, PAmount, TSpeed, PSpeed)

FOR I = 1 TO 100: NEXT I 'Timing Delay freguently used

PRINT \# 1, "P" 
FOR I = 1 TO 100: NEXT I

INPUT \#1, Reply\$

FOR I = 1 TO 200: NEXT I

TAmount $=$ TAmount $* 10000$

PRINT \# 1, SubNum\%, 0, TAmount

FOR I = 1 TO 100: NEXT I

INPUT \#1, Reply\$

FOR I = 1 TO 200: NEXT I

TSpeed $=$ INT $($ TSpeed $/ .0072)$

PRINT \#1, SubNum\%, 1, TSpeed

FOR I = 1 TO 100: NEXT I

INPUT \#1, Reply\$

FOR I = 1 TO 200: NEXT I

PAmount $=$ PAmount $* 10000$

PRINT \#1, SubNum\%, 2, PAmount

FOR I = 1 TO 100: NEXT I

INPUT \#1, Reply\$

FOR I = 1 TO 200: NEXT I

PSpeed $=$ INT(PSpeed $/ .0072)$

PRINT \#1, SubNum\%, 3, PSpeed

FOR I = 1 TO 100: NEXT I

INPUT \#1, Reply\$

FOR I = 1 TO 100: NEXT I

END SUB

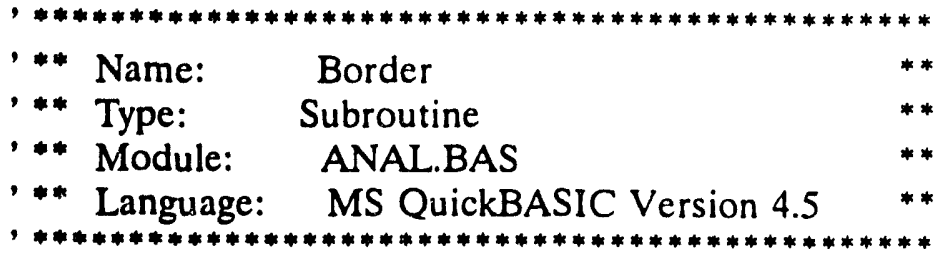

' Draws a Border about screen perimeter

' EXAMPLE OF USE: Border Green, Blue

'PARAMETERS: Clr1\% Foreground Color

' MODULE LEVEL.

$$
\text { Clr2\% Background Color }
$$

'DECLARATIONS: DECLARE SUB Border (Clr1\%, Clr2\%)

SUB Border (Clr1\%, Clr2\%)

COLOR Clr $1 \%$, Clr $2 \%$

Top\% $=1:$ Bottom $\%=25$

LOCATE Top\%, 1

PRINT SPACE\$(1); CHR\$(201);

FOR I\% = 3 TO 78: PRINT CHR\$(205); : NEXT I\%

PRINT CHR\$(187); SPACE\$(1)

FOR I\% $=$ Top $\%+1$ TO Bottom $\%-1$

LOCATE I\%, 1

PRINT SPACE\$(1); CHR\$(186); SPACE\$(1);

LOCATE I\%, 78

PRINT SPACE\$(1); CHR\$(186); SPACE\$(1);

NEXT I\%

LOCATE Bottom\%, 1

PRINT SPACE\$(1); CHR\$(200); 
FOR I\% = 3 TO 78: PRINT CHR\$(205); : NEXT I\%

PRINT CHR\$(188); SPACE\$(1);

END SUB

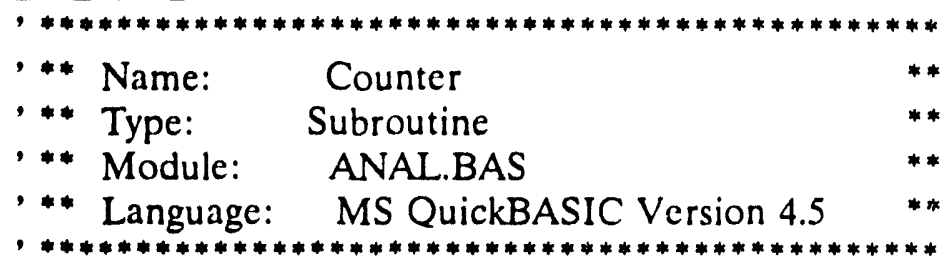

' Reads the number of counts from a counter

' EXAMPLE OF USE: Counter (Counts)

'PARAMETERS: Counts Decimal Number of counts.

' MODULE LEVEL

- DECLARATIONS: DECLARE SUB Counter (Counts)

SUB Counter (Counts)

DIM DataReg\%(3), PortValue\%(3)

ConReg\% $=\& \mathrm{H} 228$

OUT ConReg\%, \&H8

DataReg\%(0) $=\&$ H 229

DataReg\%(1) $=\&$ H22A

DataReg\%(2) $=\& \mathrm{H} 22 \mathrm{~B}$

DataReg\%(3) $=\& \mathrm{H} 22 \mathrm{C}$

Port 3 .... $>$ Bit $1=$ Strobe

,

Bit 2 = Preset

,

Bit $3=$ Reset

,

H0 Zeros

H1 Halts and Zeros

,

H4 Halts and Reads

H5 Halts Without Read

,

OUT DataReg\%(3), \&H7

H7 Starts and Restarts Without Zeroing or Reading

LOCATE 12, 24: PRINT " Counting ......... ";

DO:

PortValue\%(2) = INP(DataReg\%(2))

Value $\$=$ MID\$(HEX\$(PortValue\%(2)), 1, 1)

LOOP UNTIL Value $\$=" 7 "$

OUT DataReg\%(3), \&H4

FOR I $=0$ TO 2

PortValue\%(I) $=\operatorname{INP}($ DataReg\%(I) $)$

NEXT I

IF MID\$(HEX\$(PortValue\%(2)), 1, 1) < "8" THEN 'Correrts for Pin 47 Being PortValue\%(2) $=$ PortValue\%(2) +128 END IF

' Read Counter for Preset

FOR I = 2 TO 0 STEP -1

FOR J $=1$ TO 2

Value $\$=$ MID\$(HEX\$(PortValue\%(I)), J, 1)

IF Value $\$=" A "$ THEN Value $\$=" 10 "$

IF Value $\$=$ "B" THEN Value $\$=" 11 "$

IF Value $\$=" C "$ THEN Value $\$=" 12 "$

IF Value $\$=$ "D" THEN Value $\$=$ "13" 


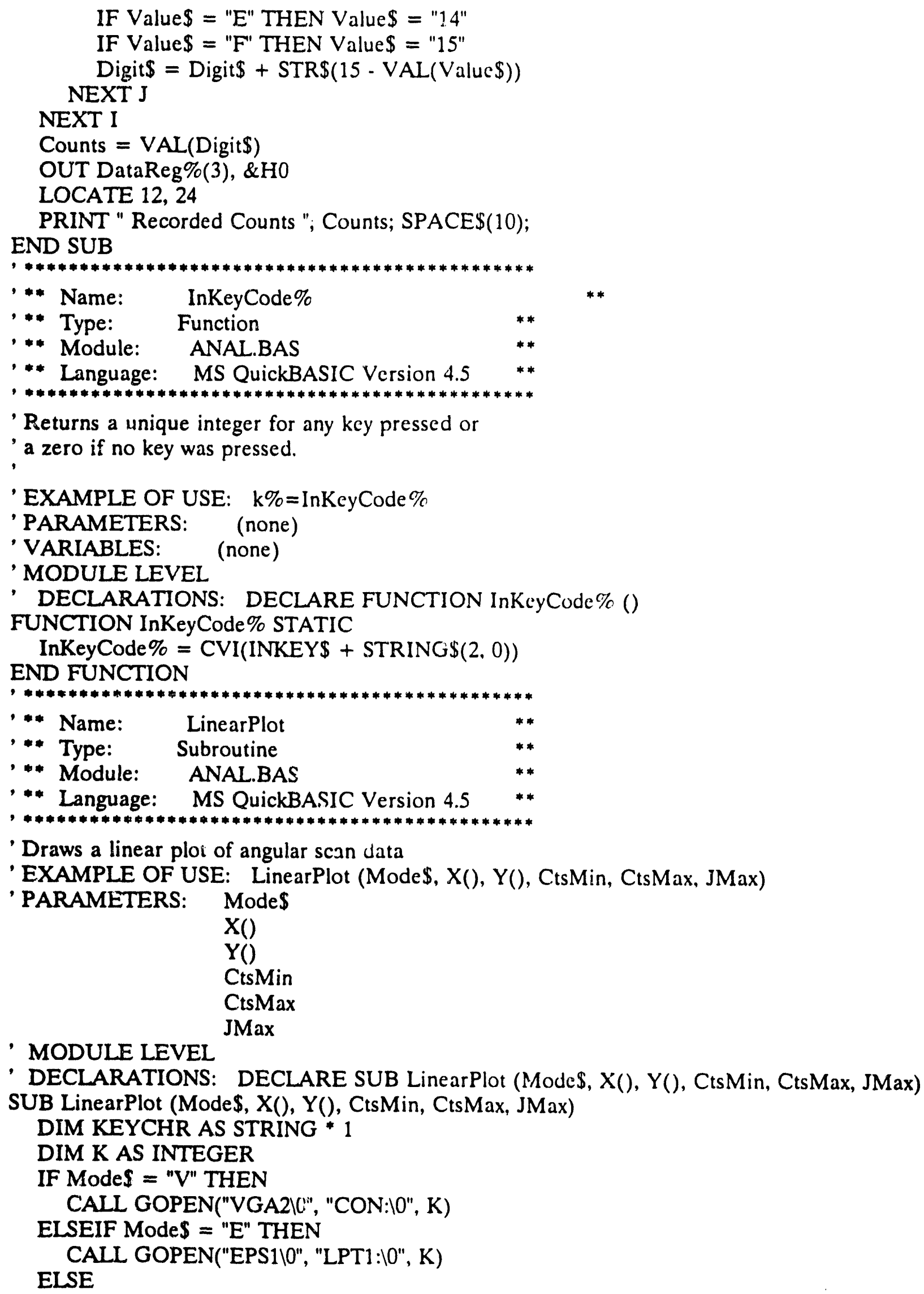




\section{EXIT SUB}

END IF

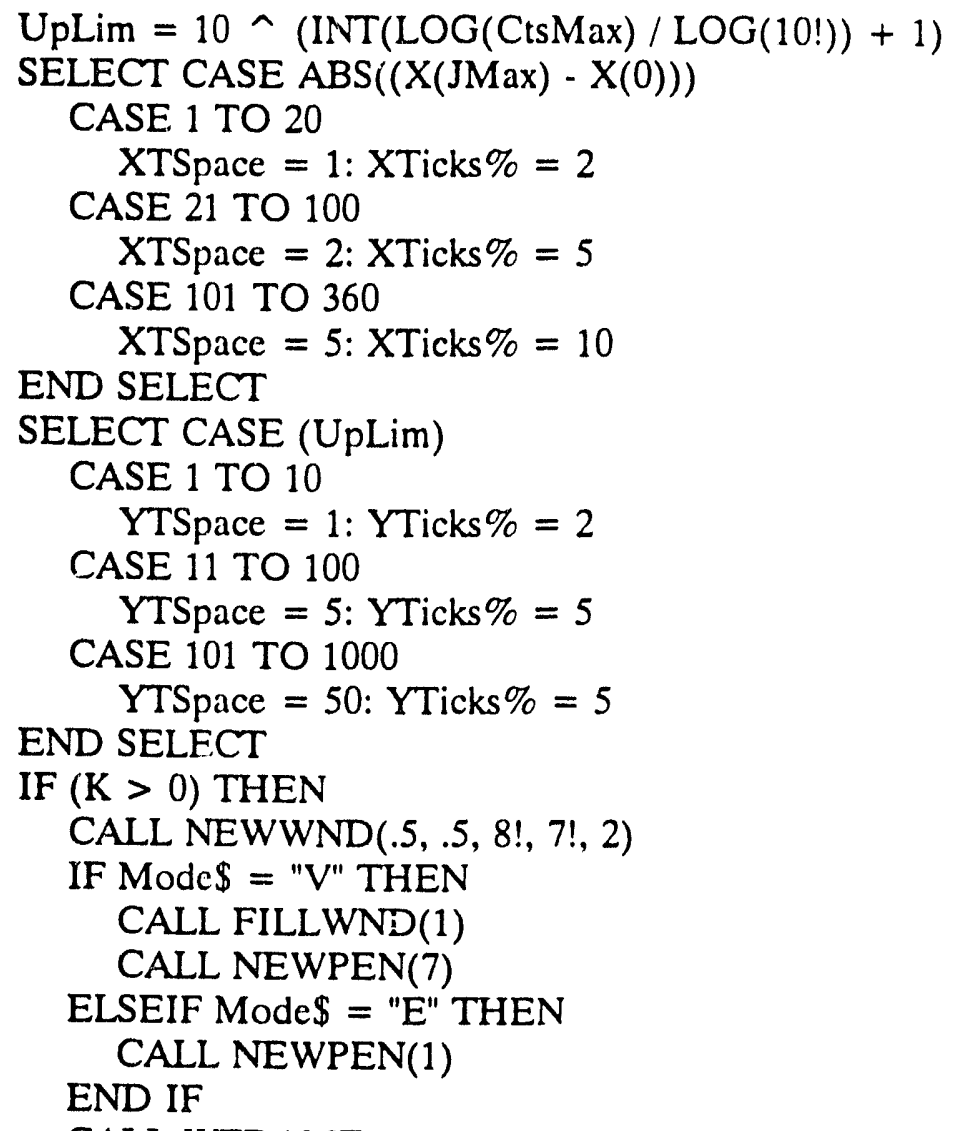

\section{CALL WFRAME}

CALL PLIMIT(1!, 1!, 6!, 5!, 2)

CALL CLIPON

CALL SETMET

CALL SCALE(X(0), X(JMax), 0, UpLim)

CALL XTSIZE $(.35, .35, .2, .2)$

CALL YTSIZE $(.35, .35, .2, .2)$

CALL MSIZE(.1)

CALL XAXIS(X(0), X(JMax), 0, XTSpace, XTicks\%)

CALL YAXIS(0, UpLim, X(0), YTSpace, YTicks\%)

CALL XAXIS(X(0), X(JMax), UpLim, XTSpace, XTicks\%)

CALL YAXIS(0, UpLim, X(JMax), YTSpace, YTicks\%)

FOR J = 0 TO JMax STEP (XTSpace * XTicks\%)

CALL MOVE $(X(J), 0)$

SELECT CASE ABS $(\mathrm{X}(\mathrm{J}))$

CASE 0 TO 9

CALL CMOVE(-1!, -2!)

CASE 10 TO 99

CALL CMOVE(-2!, -2!)

CASE 100 TO 999

CALL CMOVE(-3!, -2!)

END SELECT

CALL LABEL(STR $\$(X(J))+" \backslash 0 ")$

NEXT J 
CALL CLIPOFF

FOR J = 0 TO UpLim STEP (YTSpace * YTicks\%)

CALL MOVE $(\mathrm{X}(0), \mathrm{J})$

SELECT CASE J

CASE 0 TO 9

CALL CMOVE(-3!, 0!)

CASE 10 TO 99

CALL CMOVE(-4!, 0!)

CASE 100 TO 999

CALL CMOVE(-5!, 0!)

CASE 1000 TO 9999

CALL CMOVE(-6!, 0!)

END SELECT

CALL LABEL(STR\$(J) + "\0")

NEXT J

- CALL CLIPOFF

FOR J $=0$ TO JMax

CALL MOVE(X(J), Y(J))

CALL SETMARK(1)

CALL MARK

NEXT J

CALL GCLOSE

DO: LOOP UNTIL LEN(INKEY\$)

CALL SETTXT

\section{ELSE}

PRINT " *** DEVICE SETUP ERROR ***"

\section{END IF}

END SUB

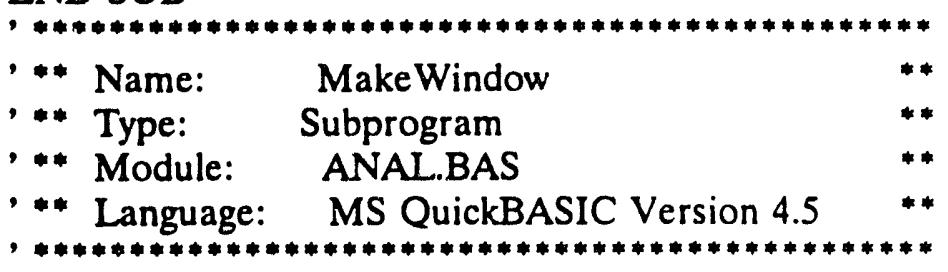

Creates a window containing a menu on the screen.

PARAMETERS: wText\$(), Row, Center\$, Column\%

wText\$(I\%) Labels to print

- Row\% Row on screen to place upper left hand corner of centered

window

- Center\$ Center box left-to-right ("C" = yes)

Column\% Column number on screen to place upper left hand corner

of ' centered window

- VARIABLES:

action -1 Create "no input" window and return

0 Create "no input" window, display and return

1 Create "active input" window, display and return

returncode choice selected (line number of wText\$ or -1 for ESC)

row row of display of upper left edge

column column of display of upper left edge

displaypage -1 for no display on this screen page

createpage screen page to create window image on 
- overpage page to overlay if multiple windows to be displayed

' MODULE LEVEL

- DECLARATIONS: DECLARE SUB MakeWindow (w AS WindowsType, wText\$(), Title\$, wPrompt\$, Center\$)

SUB MakeWindow (w AS WindowsType, wText\$(), wTitle\$, wPrompt\$, Center\$) STATIC

'Key code numbers for cursor control

CONST DOWNARROW $=20480$

CONST ENTER $=13$

CONST ESCAPE $=27$

CONST UPARROW $=18432$

CONST ENDKEY $=20224$

CONST HOMEKey $=18176$

CONST PGUP $=18688$

CONST PGDN $=20736$

CONST SPACEBAR $=32$

cursorRow\% $=$ CSRLIN

cursorCol\% $=\mathrm{POS}(0)$

SCREEN 0, 0, w.createPage, w.displayPage

IF w.screenColor > -1 THEN

COLOR White, w.screenColor: CLS

END IF

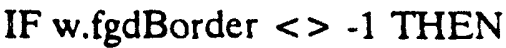

Border w.fgdBorder, w.bgdBorder

END IF

IF w.overPage $>0$ AND w.overPage $<7$ THEN

PCOPY w.overPage, w.createPage

END IF

NumStrings\% $=$ UBOUND(wText\$)

AMaxLen\% = LEN(wText\$(1))

FOR I\% $=2$ TO NumStrings\%

IF AMaxLen\% < LEN(wText\$(I\%)) THEN AMaxLen\% = LEN(wText\$(I\%)) NEXT I\%

IF UCASE $\$($ Center $\$)=$ "C" THEN

ELSE

Col\% $=37-$ INT $(($ AMaxLen\% / 2))

Col\% $=$ w.column

END IF

LOCATE w.row, Col\%

COLOR w.fgdEdge, w.bgdEdge

PRINT SPACE\$(1); CHR\$(201); STRING\$(AMaxLen\% + 4, 205); CHR\$(187); SPACE\$(1)

FOR I\% $=1$ TO NumStrings\%

LOCATE , Col\%

COLOR w.fgdEdge, w.bgdEdge

FOR $K=1$ TO 10: NEXT K

PRINT SPACE\$(1); CHR\$(186); SPACE\$(1);

COLOR w.fgdBody, w.bgdBody

PRINT SPC(1); wText\$(I\%); SPACE\$(1 + AMaxLen\% - LEN(wText\$(I\%)));

COLOR w.fgdEdge, w.bgdEdge

PRINT SPACE\$(1); CHR\$(186); SPACE\$(1);

COLOR Blue, Black: PRINT SPACE\$(2)

NEXT I\% 
LOCATE , Col\%

COLOR w.fgdEdge, w.bgdEdge

PRINT SPACE\$(1); CHR\$(200); STRING\$(AMaxLen\% + 4, 205); CHR\$(188) + SPACE\$(1);

COLOR Blue, Black: PRINT SPACE\$(2)

LOCATE , Col\% + 2

COLOR Blue, Black: PRINT SPACE $\$(A M a x L e n \%+8)$;

IF $w$.action $=1$ THEN

FOR I\% $=1$ TO NumStrings $\%$

LOCATE w.row $+I \%$, Col\% +4

COLOR Yellow, w.bgdBody

PRINT LEFT\$(wText\$(I\%), 1)

NEXT I\%

END IF

IF wTitle $\$>$ "' THEN

colTitle $\%=3+$ Col\% $+($ AMaxLen $\% / 2)-(\operatorname{LEN}(w T i t l l \$) / 2)$

LOCATE w.row, colTitle\%

COLOR w.fgdTitle, w.bgdTitle

PRINT SPACE\$(1) + wTitle\$ + SPACE\$(1):

END IF

IF wPrompt\$ $<>$ "' THEN

rowPrompt\% $=$ w.row $+1+$ NumStrings $\%$

colPrompt\% $=\mathrm{Col} \%+3+($ AMaxLen $\% / 2)-(\operatorname{LEN}($ wPrompt $\$) / 2)$

LOCATE rowPrompt $\%$, colPrompt $\%$

COLOR w.fgdPrompt, w.bgdPrompt

PRINT SPACE\$(1) + wPrompt\$ + SPACE\$(1);

END IF

IF w.action $=-1$ THEN EXIT SUB

PCOPY w.createPage, w.displayPage

SCREEN 0, 0, w.displayPage, w.displayPagc

IF $w$.action $=0$ THEN EXIT SUB

IF w.returnCode $>1$ AND w.returnCode < NumStrings $\%+1$ THEN ptr\% $=$ w.returnCode

ELSE

$\mathrm{ptr} \%=1$

END IF

IF $\operatorname{ptr} \%=1$ THEN

lastPtr\% $=2$

ELSE

last $\operatorname{Ptr} \%=\mathrm{ptr} \%-1$

END IF

choice $\$=" '$

FOR I\% = 1 TO NumStrings $\%$

tmp $\$=$ UCASE $\$(\operatorname{LTRIM} \$(w T e x t \$(I \%)))$

DO

IF tmp\$ $<>$ "' THEN

t $\$=$ LEFT $\$($ tmp $\$, 1)$

tmp\$ = MID $($ tmp\$, 2)

IF INSTR(choice $\$, \mathrm{t} \$$ ) $=0$ THEN

choice $\$=$ choice $\$+t \$$

END IF

END IF 


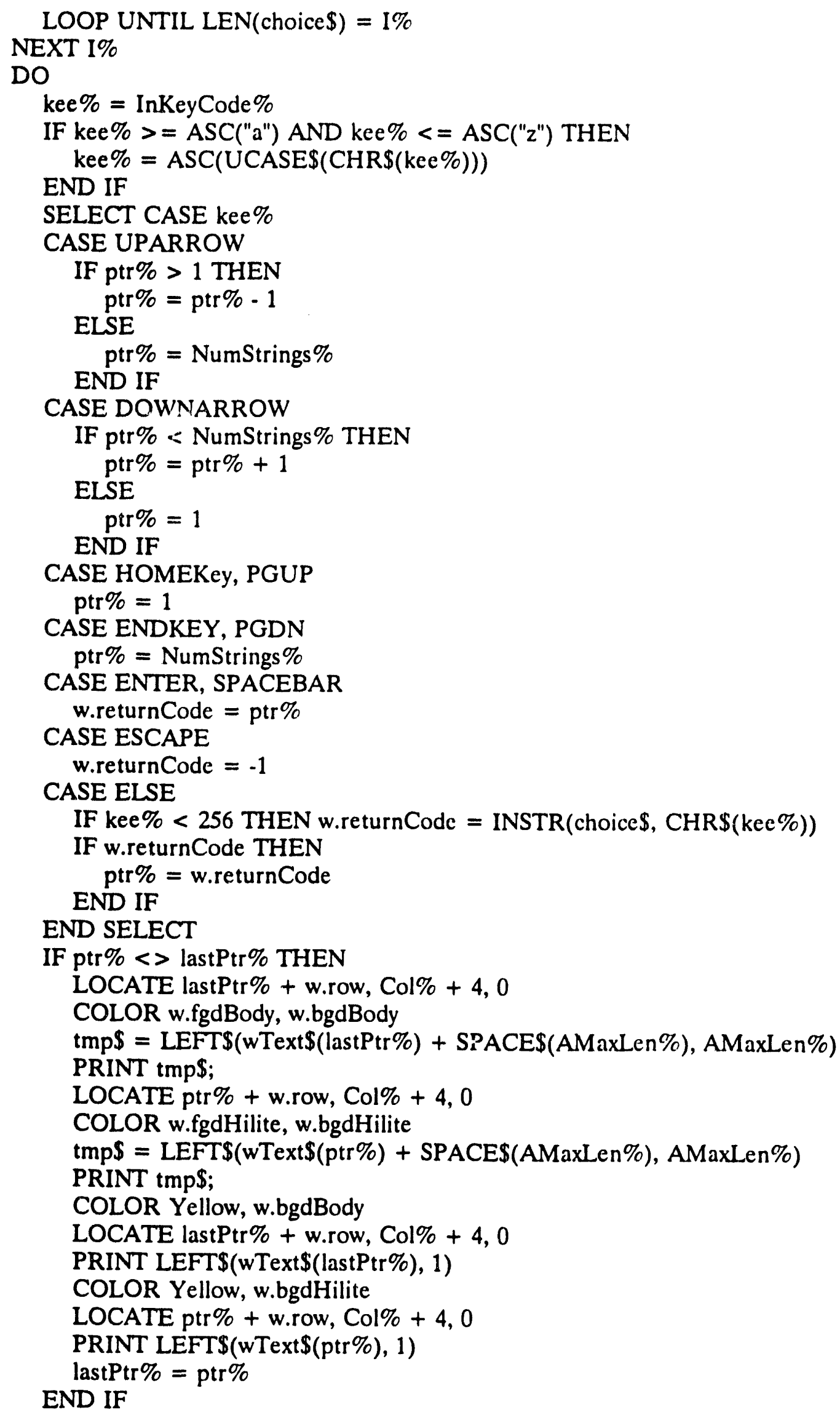


LOOP WHILE w.returnCode $=0$

LOCATE cursorRow\%, cursorCol\%

END SUB

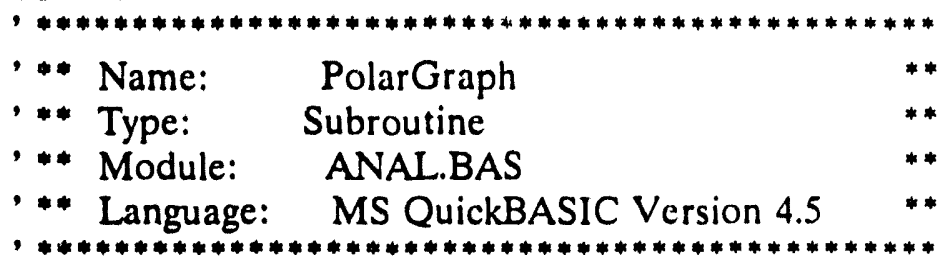

- Draws a polar plot of angular scan data

' EXAMPLE OF USE: PolarGraph (Mode\$, NPts, Th, X(), Y())

' PARAMETERS: Mode\$

$$
\begin{aligned}
& \text { X() } \\
& \text { Y() } \\
& \text { CtsMin } \\
& \text { CtsMax } \\
& \text { JMax }
\end{aligned}
$$

' MODULE LEVEL

- DECLARATIONS: DECLARE SUB PolarGraph (Mode\$, NPts, Th, X(), Y())

SUB PolarGraph (Mode\$, NPts, Th, X(), Y())

DIM KEYCHR AS STRING * 1

DIM K AS INTEGER

IF Mode $\$=$ "V" THEN

CALL GOPEN("VGA210", "CON:10", K)

ELSEIF Mode $\$=$ "E" THEN

CALL GOPEN("EPS110", "LPT1:10", K)

ELSE

EXIT SUB

END IF

$\mathrm{Th}=\mathrm{Th} / 8$ !

IF $(K>0)$ THEN

CALL NEWWND(1.25, .1, 7.25, 7.25, 2)

IF Mode $\$=$ "V" THEN

CALL FILLWND(1)

CALL NEWPEN(7)

ELSEIF Mode $\$=$ "E" THEN

CALL NEWPEN(1)

END IF

CALL WFRAME

CALL HGLTYPE(0)

CALL VGLTYPE(0)

IF Mode $\$=$ "V" THEN

CALL PLGRID(3!)

CALL NEWPEN(13)

ELSEIF Mode $\$=$ "E" THEN

CALL PRGRID(3!)

CALL NEWPEN(1)

END IF

CALL CSIZE $(.35, .35)$

CALL MSIZE (.4)

FOR I = 1 TO NPts 
CALL MOVE(Th, X(I))

CALL SETMARK(4)

CALL MARK

CALL SETMARK(1)

CALL MARK

NEXT I

IF Mode $\$=$ "V" THEN

CALL NEWPEN(10)

END IF

FOR $J=1$ TO NPts

CALL MOVE(Th, $X(J))$

CALL NLABEL(Y(J), 2)

NEXT J

CALL SETSU

FOR $J=1$ TO (NPts / 2)

CALL MOVE(Th, $X(J))$

CALL GLINE(Th, X(J + (NPts / 2)))

NEXT J

CALL GCLOSE

CALL RDKEY(KEYCHR, K)

CALL SETTXT

SCREEN 0, 0, 7, 7

COLOR BrightWhite, Cyan: CLS

ELSE

PRINT " *** DEVICE SETUP ERROR ***"

END IF

END SUB

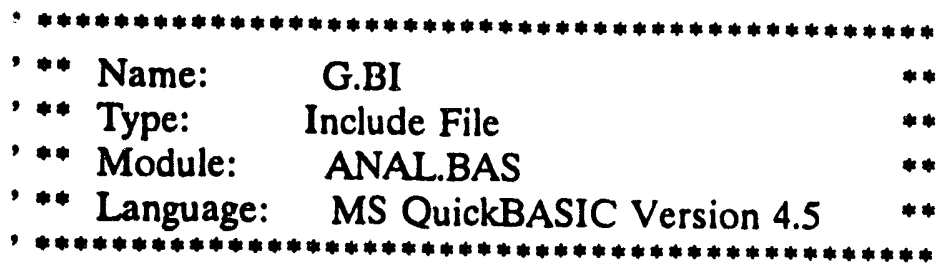

' Included File of constants and declarations

' EXAMPLE OF USE: ' \$INCLUDE: 'C:|QB45|qbfiles/G.BI'

CONST Black $=0$

CONST Blue $=1$

CONST Green $=2$

CONST Cyan $=3$

CONST Red $=4$

CONST Magenta $=5$

CONST Brown $=6$

CONST White $=7$

CONST Grey $=8$

CONST LightBlue $=9$

CONST LightGreen $=10$

CONST LightCyan $=11$

CONST LightRed $=12$

CONST LightMagenta $=13$

CONST Yellow $=14$

CONST BrightWhite $=15$ 
TYPE WindowsType

action AS INTEGER

returnCode AS INTEGER

row AS INTEGER

column AS INTEGER

bgdEdge AS INTEGER

fgdEdge AS INTEGER

fgdBody AS INTEGER

bgdBody AS INTEGER

fgdTitle AS INTEGER

bgdTitle AS INTEGER

fgdPrompt AS INTEGER

bgdPrompt AS INTEGER

fgdHilite AS INTEGER

bgdHilite AS INTEGER

fgdBorder AS INTEGER

bgdBorder AS INTEGER

screenColor AS INTEGER

displayPage AS INTEGER

createPage AS INTEGER

overPage AS INTEGER

END TYPE

TYPE Allen

Phi AS LONG

Theta AS LONG

DummyPhi AS LONG

DummyTheta AS LONG

END TYPE

- Subroutines outside of library that are part of main code

DECLARE SUB ArbMotion (SubNum\%, TAmount, PAmount, TSpeed, PSpeed)

DECLARE SUB Border (Clr1\%, Clr2\%)

DECLARE SUB Counter (Counts)

DECLARE SUB PolarGraph (Mode\$, NumP, Theta, X(), Y())

DECLARE SUB MakeWindow (w AS WindowsType, wText\$(), wTitle\$, wPrompt\$, Center\$)

DECLARE SUB LinearPlot (Mode\$, X(), Y(), CtsMin, CtsMax, JMax)

DECLARE FUNCTION InKeyCode\% ()

- SubRoutine declarations for INGRAF Library

DECLARE SUB CLIPOFF ()

DECLARE SUB CLIPON ()

DECLARE SUB CMOVE (XCHAR AS SINGLE, YCHAR AS SINGLE)

DECLARE SUB CSIZE (CW AS SINGLE, CH AS SINGLE)

DECLARE SUB FILIWND (ICOLOR AS INTEGER)

DECLARE SUB GCLOSE ()

DECLARE SUB GLINE (XPOINT AS SINGLE, YPOINT AS SINGLE)

DECLARE SUB GMOVE (XLENTH AS SINGLE, YLENTH AS SINGLE, IUNITS AS INTEGER)

DECLARE SUB GOPEN (GDNAME AS STRING, PFILE AS STRING. ISTAT AS INTEGER) DECLARE SUB GRID (XGSPC AS SINGLE, YGSPC AS SINGLE, XTSPC AS SINGLE, YTSPC AS SINGLE, XMAJCT AS INTEGER, YMAJCT AS INTEGER)

DECLARE SUB HGLTYPE (HLCODE AS INTEGER)

DECLARE SUB LABEL (LBLSTR AS STRING) 
DECLARE SUB LBLDIR (DANGLE AS SINGLE)

DECLARE SUB LBLORG (ORGCOD AS INTEGER)

DECLARE SUB LTYPE (LCODE AS INTEGER)

DECLARE SUB MARK ()

DECLARE SUB MOVE (XVAL AS SINGLE, YVAL AS SINGLE)

DECLARE SUB MSIZE (MRKSIZ AS SINGLE)

DECLARE SUB NEWPEN (PENNO AS INTEGER)

DECLARE SUB NEWWND (WX1 AS SINGLE, WY1 AS SINGLE, XLENTH AS SINGLE, YLENTH AS SINGLE, IUNITS AS INTEGER)

DECLARE SUB NLABEL (FP AS SINGLE, NFRAC AS INTEGER)

DECLARE SUB PGRID (RADVAL AS SINGLE)

DECLARE SUB PLGRID (RADIUS AS SINGLE)

DECLARE SUB PLIMIT (XREF AS SINGLE, YREF AS SINGLE, XLENTH AS SINGLE, YLENTH AS SINGLE, IUNITS AS INTEGER)

DECLARE SUB PRGRID (RADVAL AS SINGLE)

DECLARE SUB RDKEY (KEYCHAR AS STRING, KEYCOD AS INTEGER)

DECLARE SUB SETGU ()

DECLARE SUB SETMET ()

DECLARE SUB SETMARK (MARKER AS INTEGER)

DECLARE SUB SETSU ()

DECLARE SUB SETTXT ()

DECLARE SUB VGLTYPE (VLCODE AS INTEGER)

DECLARE SUB WFRAME ()

DECLARE SUB XAXIS (XMIN AS SINGLE, XMAX AS SINGLE. YINT AS SINGLE, TICSPC AS SINGLE, MAJCT AS INTEGER)

DECLARE SUB XGRID (XGSPC AS SINGLE, XTSPC AS SINGLE. MAJCT AS INTEGER) DECLARE SUB XTSIZE (PMAJ AS SINGLE, NMAJ AS SINGLE, PMIN AS SINGLE, NMIN AS_SINGLE)

DECLARE SUB YAXIS (YMIN AS SINGLE, YMAX AS SINGLE, XINT AS SINGLE, TICSPC AS SINGLE, MAJCT AS INTEGER)

DECLARE SUB YGRID (YGSPC AS SINGLE, YTSPC AS SINGLE, MAJCT AS INTEGER) DECLARE SUB YTSIZE (PMAJ AS SINGLE, NMAJ AS SINGLE, PMIN AS SINGLE, NMIN AS_SINGLE) 
ORNL/TM-12645

Dist. Category UC-25

\section{INTERNAL DISTRIBUTION}

1-2. Central Research Library

3. Document Reference Section

4-5. Laboratory Records Department

6. Laboratory Records, ORNL RC

7. ORNL Patent Section

8-10. M\&C Records Office

11-15. W. R. Allen

16. B. R. Appleton

17. C. A. Baldwin

18. L. L. Horton

19. K. J. Kozaczek
20. M. B. Lewis

21. G. M. Ludtka

22. L. K. Mansur

23. R. M. Moon

24. E. D. Specht

25. L. J. Turner

26. H. W. Foglesong (Consultant)

27. E. L. Menger (Consultant)

28 J. G. Simon (Consultant)

29. K. E. Spear (Consultant)

\section{EXTERNAL DISTRIBUTION}

30. DOE, OAK RIDGE OPERATIONS OFFICE, P.O. Box 2001, Oak Ridge, TN 37831-8600

Assistant Manager for Energy Research and Development

31-32. DOE, OFFICE OF SCIENTIFIC AND TECHNICAL INFORMATION, Office of Information Services, P.O. Box 62, Oak Ridge, TN 37831

For distribution by microfiche as shown in DOE/OSTI-4500

Distribution Category UC-25 (Materials) 

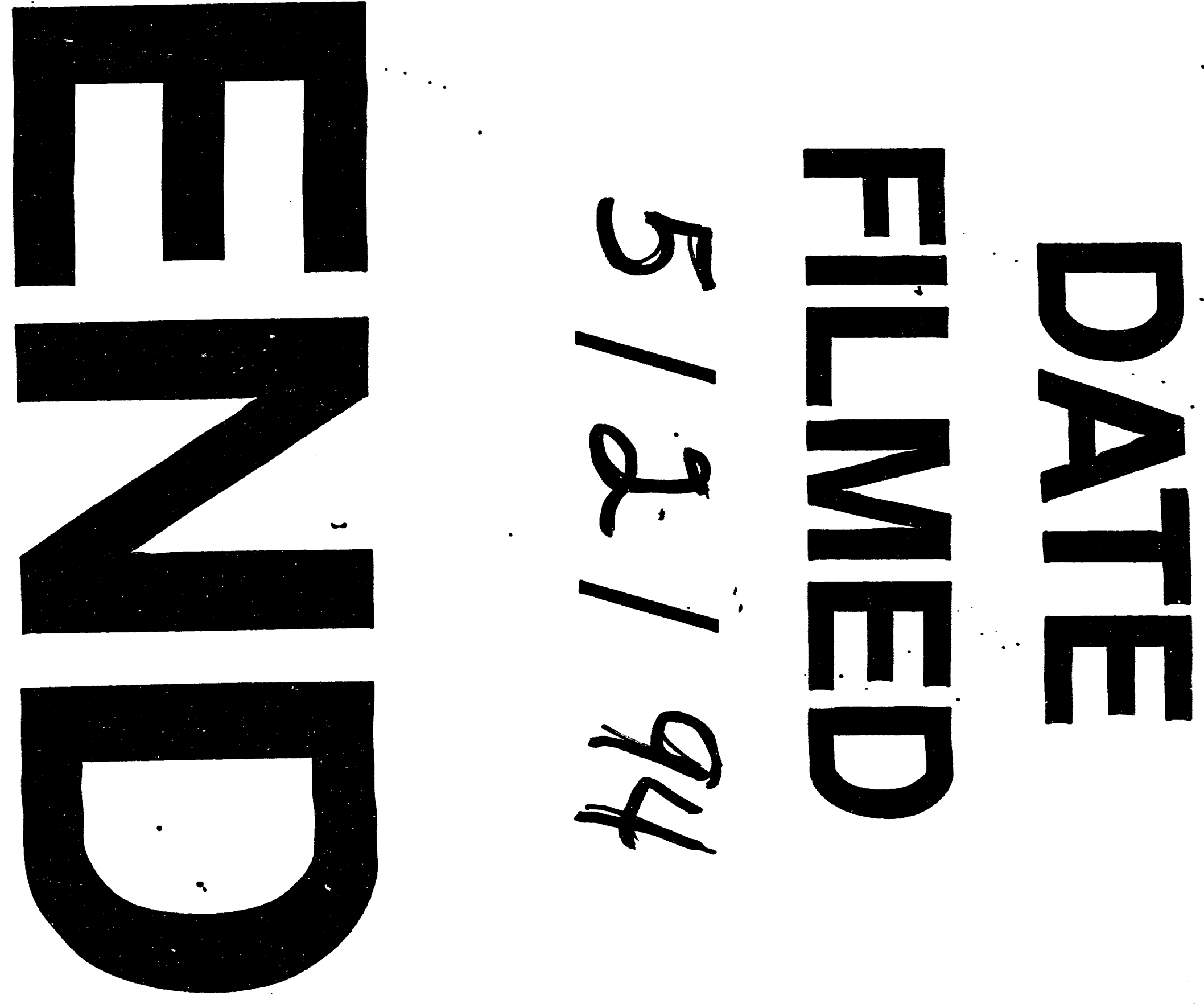
